\title{
On nonlinear Schrödinger equations in exterior domains
}

\section{Équations de Schrödinger non linéaires dans des domaines extérieurs}

\author{
N. Burq, P. Gérard*, N. Tzvetkov \\ Université Paris Sud, Mathématiques, bât 425, 91405 Orsay cedex, France \\ Received 13 December 2002; received in revised form 4 March 2003; accepted 7 March 2003
}

Available online 4 October 2003

\begin{abstract}
We prove a local smoothing effect and Strichartz type estimates for the Schrödinger equation on the exterior of a nontrapping obstacle. As a consequence we deduce global existence and uniqueness results for the Cauchy problem for nonlinear Schrödinger equations in these particular geometries.

C) 2004 L'Association Publications de l'Institut Henri Poincaré. Published by Elsevier B.V. All rights reserved

Résumé

On démontre un effet de régularisation local et des inégalités de type Strichartz pour l'équation de Schrödinger à l'extérieur d'un obstacle non captant. On en déduit des résultats d'existence globale et d'unicité pour l'équation de Schrödinger non linéaire.

(C) 2004 L'Association Publications de l'Institut Henri Poincaré. Published by Elsevier B.V. All rights reserved

MSC: 35Q55; 35BXX; 37K05; 37L50; 81Q20
\end{abstract}

Keywords: Nonlinear Schrödinger; Smoothing effect; Non-trapping; Dispersive equations

\section{Introduction}

Let $\Theta \subset \mathbb{R}^{d}, d \geqslant 2$, be a compact smooth obstacle. Denote by $\Omega$ the complementary of $\Theta$. In this paper we shall suppose that the obstacle $\Theta$ is non-trapping which means that any light ray reflecting on the boundary of $\Theta$ according to the laws of the geometric optics leaves any compact set in finite time. In other words any generalized bicharacteristic in the boundary cotangent bundle ${ }^{b} T^{*} \Omega$ (see Melrose and Sjöstrand [23,24] for a precise definition)

\footnotetext{
* Corresponding author.

E-mail addresses: Nicolas.burq@ math.u-psud.fr (N. Burq), Patrick.gerard@math.u-psud.fr (P. Gérard), Nikolay.tzvetkov@math.u-psud.fr (N. Tzvetkov).

URLs: http://www.math.u-psud.fr/ burq, http://www.math.u-psud.fr/ tzvetkov.
} 
leaves any compact set in finite time. Our goal here is to study the existence of global strong solutions for the nonlinear Schrödinger equation, posed on $\Omega$,

$$
\left(i \partial_{t}+\Delta\right) u=F(u), \quad \text { in } \mathbb{R} \times \Omega
$$

with initial data

$$
u(0, x)=u_{0}(x), \quad x \in \Omega,
$$

subject to Dirichlet boundary conditions

$$
u(t, x)=0, \quad(t, x) \in \mathbb{R} \times \partial \Omega .
$$

The nonlinear interaction $F$ is supposed to be of the form $F=\partial V / \partial \bar{z}$ with $F(0)=0$, where the "potential" $V$ is real valued and satisfies $V\left(\mathrm{e}^{i \theta} z\right)=V(z)$ for every $z \in \mathbb{C}, \theta \in \mathbb{R}$. Moreover we suppose that $V$ is of class $C^{3}$ and

$$
\left|D_{z, \bar{z}}^{k} V(z)\right| \leqslant C_{k}(1+|z|)^{2+\alpha-k}, \quad k=0,1,2,3 .
$$

Some phenomena in Physics turn out to be modeled by exterior problems and moreover one may expect rich dynamics under various boundary conditions. A first step in that direction is to establish well defined dynamics in the natural spaces determined by the conservation laws associated to (1.1). If $u(t, \cdot) \in H_{0}^{1}(\Omega) \cap H^{2}(\Omega)$ is a solution of (1.1) then (see Cazenave [13, Theorem 4.1.1]) it enjoys the conservation laws

$$
\begin{aligned}
& \frac{\mathrm{d}}{\mathrm{d} t} \int_{\Omega}|u(t, x)|^{2} \mathrm{~d} x=0 \quad \text { (charge conservation), } \\
& \frac{\mathrm{d}}{\mathrm{d} t}\left\{\int_{\Omega}|\nabla u(t, x)|^{2} \mathrm{~d} x+\int_{\Omega} V(u(t, x)) \mathrm{d} x\right\}=0 \quad \text { (energy conservation) }
\end{aligned}
$$

and therefore one can obtain via the Gagliardo-Nirenberg inequalities that for a large class of potentials $V$ the quantity $\|u(t, \cdot)\|_{H_{0}^{1}(\Omega)}$ remains finite along the trajectory starting from $u_{0} \in H_{0}^{1}(\Omega) \cap H^{2}(\Omega)$. This fact makes the study of (1.1) in the space $H_{0}^{1}(\Omega)$ of particular interest and motivates us to call $H_{0}^{1}(\Omega)$ the energy space for (1.1). It is clearly also of interest to study of $(1.1)$ in $L^{2}(\Omega)$, the space associated to the conservation law (1.4a). The main issue in the analysis is that the regularities of $H^{1}$ or $L^{2}$ are a priori too poor to be achieved by the "classical methods" (see, e.g., Segal [26], Lions [22]) for establishing local existence and uniqueness for (1.1)-(1.2)-(1.3).

The Cauchy problem associated to (1.1) with $\Omega=\mathbb{R}^{d}$ attracted much attention during last 20 years (see the books by Bourgain [3], Cazenave [13], Sulem and Sulem [27] and the references therein) and the theory of existence of finite energy (or $L^{2}$ ) solutions to (1.1) for potentials $V$ of polynomial growth has been much developed (for a discussion on this issue and open problems we refer to Bourgain [4]). Roughly speaking the argument for establishing finite energy solutions of (1.1) consists of combining $H^{1}$ local well-posedness with conservation laws (1.4a), (1.4b) which eventually provide a control on the $H^{1}$ norm. The local well-posedness is carried out by the classical Picard iteration scheme and the nonlinearity is controlled in the iteration process due to some smoothing properties of the free evolution. In the case $\Omega=\mathbb{R}^{d}$ the crucial fact on the free evolution is the family of so called Strichartz estimates which can be deduced from an explicit formula for the free solution and the Tomas-Stein restriction argument from harmonic analysis. Unfortunately in the case of exterior problem no suitable explicit representation of the free evolution is available and therefore the problem of establishing Strichartz estimates for the solution of (1.1) with $F=0$ meets serious difficulties. However as it was shown by our experience with NLS on compact manifolds (see [9]) one may approach the problem of the existence of finite energy solutions for (1.1) even with weaker linear estimates than the whole family of Strichartz inequalities. That is exactly what we are going to do here.

In $2 d$, local well-posedness in $H_{D}^{s}(\Omega)$ (see the next section for definition of that space), $s>1$, for the initial boundary value problem (1.1)-(1.2) - (1.3) can be obtained by "classical methods" and therefore one barely misses the key regularity $H^{1}$. Nevertheless it is known that for $\alpha \leqslant 2$ (see Cazenave [13, Theorem 4.5.1], Brézis and 
Gallouet [5], Vladimirov [38], Ogawa and Ozawa [25]) one can obtain the global existence of $H^{1}$ solution to (1.1) for a suitable class of potentials $V$. The work of M. Tsutsumi [32] shows that one could extend the result to $\alpha \in] 2,3]$ if the data $u_{0} \in H_{0}^{1}(\Omega)$ is such that such that $\Delta u_{0} \in H_{0}^{1}(\Omega)$. Here we will be able to extend these results to much more general nonlinearities. Even when $\alpha \leqslant 3$ we have a stronger result comparing to the above mentioned works since we obtain that the flow map is Lipschitz continuous on bounded sets of $H_{0}^{1}(\Omega)$. On the other hand the considerations in $[5,38,25,32]$ are valid on any domain $\Omega$ with smooth boundary without any geometric assumption.

The main difficulty in higher dimensions is that one needs to "gain at least $1 / 2$ derivative" with respect to the classical well-posedness results. We will be able to do this as far as $\alpha<\frac{2}{d-2}$ which does not cover all possible nonlinearities for $H^{1}$ theory in the case $\Omega=\mathbb{R}^{d}$. Recall that (see, e.g., Kato [18]) when $\Omega=\mathbb{R}^{d}$ the critical order of the nonlinearity for the well-posedness in the energy space $H^{1}$ turns out to be $\alpha=\frac{4}{d-2}$. It seems however that here we obtain the first global existence and uniqueness results in dimensions $d \geqslant 3$ for (1.1)-(1.2)-(1.3) with large initial data. It should be mentioned that "small data techniques" can be applied to (1.1)-(1.2)-(1.3) under some geometric assumptions (which imply our non-trapping assumption) on $\Theta$ (see Y. Tsutsumi [34], M. Tsutsumi [33]). That approach yields the global existence of small amplitude solutions to (1.1)-(1.2)-(1.3) in any dimension for nonlinearities of sufficiently high order (and initial data sufficiently smooth).

We now state our result concerning finite energy solutions.

Theorem 1. Suppose that $\alpha<\frac{2}{d-2}, V(z) \geqslant-C(1+|z|)^{\beta}, \beta<2+\frac{4}{d}$ and that $\Theta$ is non-trapping. Then

(1) For any $u_{0} \in H_{0}^{1}(\Omega)$ the initial boundary value problem (1.1)-(1.2)-(1.3) has a unique global solution $u \in C\left(\mathbb{R} ; H_{0}^{1}(\Omega)\right)$ satisfying the conservation laws $(1.4 \mathrm{a}),(1.4 \mathrm{~b})$.

(2) If $d=2,3,4$, for any $T>0$ the flow map $u_{0} \mapsto u$ is Lipschitz continuous from any bounded set of $H_{0}^{1}(\Omega)$ to $C\left([-T, T] ; H_{0}^{1}(\Omega)\right)$.

(3) When $d=3$ and $\alpha=2$ statements (1) and (2) hold provided $\left\|u_{0}\right\|_{H_{0}^{1}(\Omega)}$ be sufficiently small.

Our proof of Theorem 1 strongly relies on a local smoothing effect for the free evolution $\exp \left(\right.$ it $\left.\Delta_{D}\right)$, where $\Delta_{D}$ is the Laplace operator acting on $L^{2}(\Omega)$, with domain $D=H^{2}(\Omega) \cap H_{0}^{1}(\Omega)$. This phenomenon has been first observed in the case of $\mathbb{R}^{d}$ in the works of Constantin and Saut [14], Sjölin [28] and Vega [37]. It was later generalized by many authors to different perturbations of the flat Laplacian (see Ben Artzi and Klainerman [1], Constantin and Saut [15], Doi [17] ... ). It is important to realize that the local smoothing can be reduced to bounds on the cut-off resolvent of the corresponding stationary operator. Since such resolvent estimates are fortunately available for the exterior problem of non-trapping obstacle we will be able in Section 2 below to derive a local smoothing estimate for $\exp \left(i t \Delta_{D}\right)$ and hence to extend the above mentioned results to the case of boundary value problems, a fact which seems to be of independent interest. Following a strategy suggested by Staffilani and Tataru [30], we shall also be able to prove that away from the obstacle the free evolution enjoys the Strichartz estimates exactly as for the flat space. Once we have the linear estimates we perform the usual Picard iteration to get $H^{1}$ well-posedness for the nonlinear problem. Let us mention that the assumption $V(z) \geqslant-C(1+|z|)^{\beta}$ in Theorem 1 is crucial for the global existence of solutions. For example, if $\alpha=2, d=2$ and $V(z)=-|z|^{4}$, regular solutions can develop singularities in finite time (see [11], Remark 1.1). Blow up phenomena for boundary problems with more general nonlinearities are displayed in Kavian [19] by using viriel type identities, however it is not clear to us whether these arguments can be applied to exterior domains. Note that despite of the fact that the functional $F$ is not Lipschitz continuous on bounded sets of $H_{0}^{1}(\Omega)$, due to the "dispersive properties" of the linear part of the equation, the flow map turns out to have that property at least for $d \leqslant 4$. It is an interesting problem to check that property in dimensions higher than 4.

Our second global well-posedness result deals with $L^{2}$ solutions. 
Theorem 2. Suppose that $\alpha<2 / d$ and that $\Theta$ is non-trapping. Then for any $u_{0} \in L^{2}(\Omega)$ the initial boundary value problem (1.1)-(1.2)-(1.3) has a unique global solution in the following class $X$ :

- If $\alpha<\frac{1}{d}$ then $X=C\left(\mathbb{R} ; L^{2}(\Omega)\right)$.

- If $\alpha \geqslant \frac{1}{d}$ then $X$ is any of the spaces $C\left(\mathbb{R} ; L^{2}(\Omega)\right) \cap L_{\mathrm{loc}}^{p}\left(\mathbb{R} ; L^{q}(\Omega)\right)$ where $(p, q)$ satisfy $\frac{1}{p}+\frac{d}{q}=\frac{d}{2}$, $2<p<\frac{2(\alpha+1)}{\alpha d-1}$.

Moreover

(1) The solution $u$ satisfies the conservation law (1.4a).

(2) For any pair $(p, q)$ satisfying $2<p \leqslant \infty, \frac{1}{p}+\frac{d}{q}=\frac{d}{2}$ one has $u \in L_{\mathrm{loc}}^{p}\left(\mathbb{R} ; L^{q}(\Omega)\right)$.

(3) For any $T>0$ the flow map $u_{0} \mapsto u$ is Lipschitz continuous from any bounded set of $L^{2}(\Omega)$ to $C\left([-T, T] ; L^{2}(\Omega)\right)$.

Remark 1.1. The result of Theorem 2 is in strong contrast with the case of a bounded open set $\Omega$. Indeed, in [12], we proved that, if $\Omega$ is a ball, there exists some $\alpha_{0}>0$ such that, for every $\left.\left.\alpha \in\right] 0, \alpha_{0}\right]$, the Cauchy problem for

$$
i \partial_{t} u+\Delta_{D} u=\left(1+|u|^{2}\right)^{\alpha / 2} u
$$

is not well-posed on $L^{2}(\Omega)$ in the sense of Theorem 2 .

Remark 1.2. For the sake of conciseness, we have chosen to restrict the study to the case of Dirichlet boundary conditions. However, the case of Neumann conditions could be handled using the same ideas (see Remarks 2.3 and 2.9).

Remark 1.3. The structural assumptions on the nonlinear interaction $F$ are needed to establish the global wellposedness. If one is interested only in local in time results then we can assume only the following growth conditions,

$$
\begin{aligned}
& \left|F\left(z_{1}\right)-F\left(z_{2}\right)\right| \lesssim\left|z_{1}-z_{2}\right|\left(1+\left|z_{1}\right|+\left|z_{2}\right|\right)^{\alpha}, \\
& \left|\left(D_{z, \bar{z}} F\right)\left(z_{1}\right)-\left(D_{z, \bar{z}} F\right)\left(z_{2}\right)\right| \lesssim\left|z_{1}-z_{2}\right|\left(1+\left|z_{1}\right|+\left|z_{2}\right|\right)^{\max \{\alpha-1,0\}} .
\end{aligned}
$$

The rest of the paper is organized as follows. We complete this section by introducing some notation. In Section 2, we first state the Sobolev embeddings we need for the sequel. Then we state some estimates for the cut-off resolvent of $\Delta_{D}$. Further we prove local smoothing estimates in the form needed for the proof of the crucial nonlinear estimate. We complete Section 2 by proving Strichartz type inequalities for $\exp \left(\right.$ it $\left.\Delta_{D}\right)$. We distinguish the cases when we evaluate the free wave away from the obstacle. Section 3 is devoted to the proof of Theorem 1 while Section 4 deals with the proof of Theorem 2.

Notations. For $T>0, p \in[1,+\infty]$, if $X$ is a Banach space, we denote by $L_{T}^{p} X$ the Banach space of $X$ valued functions on $[0, T]$ equipped with the following norm

$$
\|f\|_{L_{T}^{p} X}=\left\{\int_{0}^{T}\|f(t)\|_{X}^{p} \mathrm{~d} t\right\}^{1 / p}
$$

with the usual modification for $p=+\infty$. For any positive $A$ and $B$ the notation $A \lesssim B$ (respectively $A \gtrsim B$ ) means that there exists a positive constant $c$ such that $A \leqslant c B$ (respectively $A \geqslant c B$ ).

\section{Linear estimates}

\subsection{Functional spaces and embeddings}

Let $\Omega \subset \mathbb{R}^{d}, d \geqslant 2$, be a smooth domain. For $s \geqslant 0, p \in[1,+\infty]$, we denote by $W^{s, p}(\Omega)$ the Sobolev spaces on $\Omega$. We write $L^{p}(\Omega)$ and $H^{s}(\Omega)$ instead of $W^{0, p}(\Omega)$ and $W^{s, 2}(\Omega)$ respectively. For $s \in \mathbb{Z}_{+}$the norm in 
$W^{s, p}(\Omega)$ can be expressed in an explicit way while for non-integer values of $s$ more care is needed and one can define in this case the spaces $W^{s, p}(\Omega)$ by suitable interpolation (see [2]). By $W_{0}^{1, q}(\Omega)$, we denote the closure of $C_{0}^{\infty}(\Omega)$ in $W^{1, q}(\Omega)$. The space $W_{0}^{1,2}(\Omega)$ is usually denoted by $H_{0}^{1}(\Omega)$. If $\Omega$ has compact boundary then we have

$$
W^{1, q}(\Omega) \cap H_{0}^{1}(\Omega) \subset W_{0}^{1, q}(\Omega), \quad q \geqslant 2 .
$$

In order to obtain (2.1), we can use that $W_{0}^{1, q}(\Omega)$ can be identified with the kernel of the natural trace map from $W^{1, q}(\Omega)$ to $L^{q}(\partial \Omega)$ and then use that $L^{q}(\partial \Omega) \subset L^{2}(\partial \Omega), q \geqslant 2$, which follows from the compactness of the boundary. By $\Delta_{D}$ we denote the Dirichlet Laplacian on $\Omega$. The domain of $\Delta_{D}$ is $H_{0}^{1}(\Omega) \cap H^{2}(\Omega)$. For $s \geqslant 0$, we can define $\left(-\Delta_{D}+1\right)^{s / 2}$ via the functional calculus of self-adjoint operators. We denote by $H_{D}^{s}(\Omega)$ the domain of $\left(-\Delta_{D}+1\right)^{s / 2}$. It is known that (see, e.g., [31])

$$
H_{D}^{1}(\Omega)=H_{0}^{1}(\Omega)
$$

and we will make often use of (2.2) without explicit mention. We next define $H_{D}^{-1}(\Omega)$ (this space is often denoted in the literature simply by $H^{-1}(\Omega)$ ) as the dual of $H_{D}^{1}(\Omega)$. Then we define $H_{D}^{s}(\Omega)$ for $s \in[-1,0]$ via interpolation and due to Corollary 4.5.2 in [2], we have the duality between $H_{D}^{s}(\Omega)$ and $H_{D}^{-s}(\Omega)$ for $s \in[0,1]$. We now state the Sobolev embeddings that will be used in that paper.

Proposition 2.1. Let $\Omega \subset \mathbb{R}^{d}, d \geqslant 2$ be smooth domain. Then the following continuous embeddings hold

$$
\begin{aligned}
& H_{0}^{1}(\Omega) \subset L^{p}(\Omega), \quad 2 \leqslant p \leqslant \frac{2 d}{d-2}(p<+\infty \text { if } d=2), \\
& H_{D}^{s}(\Omega) \subset L^{p}(\Omega), \quad \frac{1}{2}-\frac{1}{p}=\frac{s}{d}, s \in[0,1[, \\
& H_{D}^{s+1}(\Omega) \subset W^{1, p}(\Omega), \quad \frac{1}{2}-\frac{1}{p}=\frac{s}{d}, s \in[0,1[, \\
& W^{1, p}(\Omega) \subset L^{q}(\Omega), \quad \frac{1}{p}-\frac{1}{q}=\frac{1}{d}, 1 \leqslant p<q<+\infty \\
& W^{s, p}(\Omega) \subset L^{\infty}(\Omega), \quad s>\frac{d}{p}, p \geqslant 1, \\
& H_{D}^{s+\frac{1}{p}}(\Omega) \subset W^{s, q}(\Omega), \quad \frac{1}{p}+\frac{d}{q}=\frac{d}{2}, p \geqslant 2, s \in[0,1] .
\end{aligned}
$$

The proof of Proposition 2.1 follows from the standard Sobolev embeddings and the use of extension operators.

\subsection{Resolvent estimates}

Since $-\Delta_{D}$ is a positive self-adjoint operator the resolvent $\left(-\Delta_{D}-\lambda\right)^{-1}$ is analytic in $\mathbb{C} \backslash \mathbb{R}^{+}$. In this section we collect several bounds for $\left(-\Delta_{D}-\lambda\right)^{-1}$ when $\lambda$ approaches $\mathbb{R}^{+}$. We first state the high frequencies bound.

Proposition 2.2. For every $\chi \in C_{0}^{\infty}\left(\mathbb{R}^{d}\right), d \geqslant 2$, there exists a positive constant $C$ such that for every $|\lambda| \geqslant 1$ and $0<\varepsilon \ll 1$ one has

$$
\left\|\chi\left(-\Delta_{D}-(\lambda \pm i \varepsilon)^{2}\right)^{-1} \chi\right\|_{L^{2}(\Omega) \rightarrow L^{2}(\Omega)} \leqslant C|\lambda|^{-1} .
$$

The result of Proposition 2.2, for which the non-trapping assumption plays a crucial role, is proven for $|\lambda| \gg 1$ in greater generality by Lax and Phillips [21], Melrose and Sjöstrand [23,24], Vainberg [35], Vasy and Zworski [36]. We also refer to [8] for a self contained proof which, joined with the results in [6], would relax the smoothness 
assumption. The boundedness of the cut-off resolvent on $L^{2}(\Omega)$ for finite $|\lambda| \neq 0$ results from Rellich uniqueness theorem (see [21] or [7, Annexe B.1]). Proposition 2.2 can be also stated as a weighted $L^{2}$ estimate for the operator $\left(-\Delta_{D}-(\lambda \pm i \varepsilon)^{2}\right)^{-1}$.

Remark 2.3. Proposition 2.2 is also true for the resolvent associated to Neumann boundary conditions. The proof in this case is the same, using propagation of singularities arguments.

Next we state the small frequencies bound.

Proposition 2.4. Assume that $\Theta \neq \emptyset$. Then for every $\chi \in C_{0}^{\infty}\left(\mathbb{R}^{d}\right), d \geqslant 2$, the cut-off resolvent $\chi\left(-\Delta_{D}-(\lambda \pm\right.$ $\left.i \varepsilon)^{2}\right)^{-1} \chi,|\lambda| \leqslant 1,0<\varepsilon \ll 1$ is a bounded operator on $L^{2}(\Omega)$ with an operator norm independent of $\lambda$ and $\varepsilon$.

For the proof of Proposition 2.4, we refer to [7, Annexe B.2]. Remark that this latter proof breaks down if $\Theta=\emptyset$ since the Poincaré inequality is used to control the local $L^{2}$-norm of a function by the local $L^{2}$-norm of its gradient (that is is why $\Theta \neq \emptyset$ is required). Propositions 2.2, 2.4 can be used to prove the boundedness of the cut-off resolvent between Sobolev spaces as shows the next proposition.

Proposition 2.5. Assume that $\Theta \neq \emptyset$. Then for every $\chi \in C_{0}^{\infty}\left(\mathbb{R}^{d}\right), d \geqslant 2, \chi \geqslant 0$, every $s \geqslant-1$ there exists a positive constant $C$ such that for every $\lambda \in \mathbb{R}$ and $0<\varepsilon \ll 1$ one has

$$
\left\|\chi\left(-\Delta_{D}-(\lambda \pm i \varepsilon)^{2}\right)^{-1} \chi\right\|_{H_{D}^{s}(\Omega) \rightarrow H_{D}^{s+1}(\Omega)} \leqslant C .
$$

Remark 2.6. For $\operatorname{Re} z<0$, an integration by parts gives

$$
\left\|\chi\left(-\Delta_{D}-z\right)^{-1} \chi\right\|_{H_{D}^{s}(\Omega) \rightarrow H_{D}^{s+1}(\Omega)} \leqslant \frac{C}{(1+|\operatorname{Re} z|)^{1 / 2}}
$$

which, in the region $-\varepsilon^{2}<\operatorname{Re} z<0$, implies the same estimate as in (2.9) (one can get even better).

Proof of Proposition 2.5. Set $\mu=\lambda \pm i \varepsilon$ and let $u$ and $f$ be such that

$$
\left(\Delta_{D}+\mu^{2}\right) u=\chi f .
$$

We multiply (2.11) by $\chi \bar{u}$ and after integration on $\Omega$, we get

$$
-\int \chi|\nabla u|^{2}+\mu^{2} \int \chi|u|^{2}-\int(\nabla u, \nabla \chi) \chi_{1} \bar{u}=\int \chi^{2} f \bar{u}
$$

where $\chi_{1} \in C_{0}^{\infty}\left(\mathbb{R}^{d}\right), \chi_{1} \geqslant 0$, is equal to one on the support of $\chi$ and $(\cdot, \cdot)$ denotes the scalar product in $\mathbb{C}^{d}$. Since $\chi \lesssim \chi_{1}^{2}$ and using that $|\mu| \leqslant|\lambda|+1$ we obtain that for every $\delta>0$,

$$
\int \chi|\nabla u|^{2} \lesssim(|\lambda|+1)^{2} \int \chi_{1}^{2}|u|^{2}+\delta \int|\nabla \chi|^{2}|\nabla u|^{2}+(4 \delta)^{-1} \int\left|\chi_{1} u\right|^{2}+\left|\int \chi^{2} f \bar{u}\right| .
$$

Since $|\nabla \chi|^{2} \lesssim \chi$ and by choosing $\delta$ small enough, we get

$$
\int \chi|\nabla u|^{2} \lesssim(|\lambda|+1)^{2}\left\|\chi_{1} u\right\|_{L^{2}(\Omega)}^{2}+\|\chi f\|_{L^{2}(\Omega)}^{2} .
$$

Using Propositions 2.2, 2.4, we deduce that

$$
(|\lambda|+1)\left\|\chi_{1} u\right\|_{L^{2}(\Omega)} \lesssim(|\lambda|+1)\left\|\chi_{1}\left(\Delta_{D}+\mu^{2}\right)^{-1}\left(\chi_{1} \chi f\right)\right\|_{L^{2}(\Omega)} \lesssim\|\chi f\|_{L^{2}(\Omega)}
$$


and therefore

$$
\int \chi|\nabla u|^{2} \lesssim\|\chi f\|_{L^{2}(\Omega)}^{2} .
$$

Using again Propositions 2.4 and 2.2, we get

$$
\|\chi u\|_{H_{D}^{1}(\Omega)} \lesssim\|\chi f\|_{L^{2}(\Omega)}
$$

This completes the proof of Proposition 2.5 for $s=0$, i.e.,

$$
\left\|\chi\left(-\Delta_{D}-(\lambda \pm i \varepsilon)^{2}\right)^{-1} \chi\right\|_{L^{2}(\Omega) \rightarrow H_{D}^{1}(\Omega)} \leqslant C .
$$

Dualizing (2.12), we obtain,

$$
\left\|\chi\left(-\Delta_{D}-(\lambda \pm i \varepsilon)^{2}\right)^{-1} \chi\right\|_{H_{D}^{-1}(\Omega) \rightarrow L^{2}(\Omega)} \leqslant C
$$

which yields Proposition 2.5 with $s=-1$.

We next prove it for $s=1$. Let again $u$ and $f$ be such that (2.11) holds and $\chi_{1} \in C_{0}^{\infty}\left(\mathbb{R}^{d}\right), \chi_{1} \geqslant 0$, be equal to one on the support of $\chi$. Write

$$
\|\chi u\|_{H_{D}^{2}(\Omega)} \approx\|\chi u\|_{H_{D}^{1}(\Omega)}+\left\|\Delta_{D}(\chi u)\right\|_{L^{2}(\Omega)} .
$$

Since $\|\chi u\|_{H_{D}^{1}(\Omega)}$ can be estimated by means of (2.12), we only need to bound $\left\|\Delta_{D}(\chi u)\right\|_{L^{2}(\Omega)}$. Further we write

$$
\Delta_{D}(\chi u)=\chi \Delta_{D} u+\left[\Delta_{D}, \chi\right] \chi_{1} u
$$

and using that the commutator $\left[\Delta_{D}, \chi\right]$ is bounded from $H_{D}^{1}(\Omega)$ to $L^{2}(\Omega)$, we get

$$
\left\|\left[\Delta_{D}, \chi\right] \chi_{1} u\right\|_{L^{2}(\Omega)} \lesssim\left\|\chi_{1} u\right\|_{H_{D}^{1}(\Omega)}
$$

Using (2.12), we obtain

$$
\left\|\chi_{1} u\right\|_{H_{D}^{1}(\Omega)}=\left\|\chi_{1}\left(\Delta_{D}+\mu^{2}\right)^{-1}\left(\chi_{1} \chi f\right)\right\|_{L^{2}(\Omega)} \lesssim\|\chi f\|_{L^{2}(\Omega)} .
$$

It remains to bound $\left\|\chi \Delta_{D} u\right\|_{L^{2}(\Omega)}$. Since $\Delta_{D} u=\chi f-\mu^{2} u$, we deduce that $\Delta_{D} u \in H_{D}^{1}(\Omega)$. Since $\Delta_{D} u$ solves the equation

$$
\left(\Delta_{D}+\mu^{2}\right)\left(\Delta_{D} u\right)=\Delta_{D}(\chi f),
$$

a use of (2.13) yields

$$
\left\|\chi \Delta_{D} u\right\|_{L^{2}(\Omega)} \lesssim\left\|\chi_{1} \Delta_{D}(\chi f)\right\|_{H_{D}^{-1}(\Omega)} \lesssim\|\chi f\|_{H_{D}^{1}(\Omega)},
$$

where we used that $\chi_{1} \Delta_{D}$ is bounded from $H_{D}^{1}(\Omega)$ to $H_{D}^{-1}(\Omega)$. This proves the result for $s=1$. Since we obtained (2.9) for $s=-1$ and $s=1$ we can use an interpolation argument to get it for $s \in[-1,1]$. Applying the operator $\Delta_{D}$ to the equation and an induction argument give the result for any $s \in \mathbb{N}$. Finally we use interpolation to get it for any $s \geqslant 1$.

\subsection{Local smoothing}

Now we are going to use the resolvent bounds of the previous section to deduce several estimates for the linear Schrödinger equation posed on $\Omega$ with Dirichlet boundary conditions. This procedure is known in the literature at least for the homogeneous estimates (see, for example, [1]). The proof presented here is based on the observation that it is sufficient to establish the non-homogeneous bound and then all other estimates follow by the so called $T T^{\star}$ argument together with a simple symmetry consideration. Finally the nonhomogeneous estimate is proven by 
performing Fourier transform in time and applying Proposition 2.5. From now on we shall work on positive time intervals only. Of course similar considerations apply to negative time intervals.

Proposition 2.7. Assume that $\Theta \neq \emptyset$. Then for every $T>0$, for every $\chi \in C_{0}^{\infty}\left(\mathbb{R}^{d}\right), d \geqslant 2$,

$$
\|\chi u\|_{L_{T}^{2} H_{D}^{s+1}(\Omega)} \leqslant C\|\chi f\|_{L_{T}^{2} H_{D}^{s}(\Omega)},
$$

where $s \in[-1,1]$ and $u(t)=\int_{0}^{t} \mathrm{e}^{i(t-\tau) \Delta_{D}} \chi f(\tau) \mathrm{d} \tau$,

$$
\|\chi v\|_{L_{T}^{2} H_{D}^{s+1 / 2}(\Omega)} \leqslant C\left\|v_{0}\right\|_{H_{D}^{s}(\Omega)},
$$

where $s \in[0,1]$ and $v(t)=\mathrm{e}^{i t \Delta_{D}} v_{0}$.

Remark 2.8. Remark that in the estimate above $v_{0}$ is not assumed to have compact support. Remark also that the proof will show that the constants $C$ do not depend on $T$, i.e., the estimates are global in time.

Proof of Proposition 2.7. We first prove (2.14). Extend $f(\tau, \cdot)$ by zero for $\tau \notin[0, T]$. According to the support properties of $f$ and $u$ their Fourier transforms (in time) are holomorphic in the domain $\{\operatorname{Im} z<0\}$ and satisfy the equation

$$
\left(-z+\Delta_{D}\right) \hat{u}(z, \cdot)=\chi \hat{f}(z, \cdot)
$$

Taking $z=\lambda-i \varepsilon, \lambda \in \mathbb{R}, \varepsilon>0$, letting $\varepsilon$ tend to zero, using Proposition 2.5 and Remark 2.6, we get

$$
\|\chi \hat{u}\|_{L^{2}\left(\mathbb{R} ; H_{D}^{s+1}(\Omega)\right)} \lesssim\|\chi \hat{f}\|_{L^{2}\left(\mathbb{R} ; H_{D}^{s}(\Omega)\right)}, \quad s \in[-1,1] .
$$

The proof of (2.14) is completed by observing that the Fourier transform of any function from $\mathbb{R}$ to a Hilbert space $H$ defines an isometry on $L^{2}(\mathbb{R} ; H)$.

Now we turn to the proof of (2.15). We first prove it for $s=0$, i.e. if we denote by $A$ the operator which to given $u_{0} \in L^{2}(\Omega)$ associates $\chi \mathrm{e}^{i t \Delta_{D}} u_{0}$, we need to prove that $A$ is bounded from $L^{2}(\Omega)$ to $L_{T}^{2} H_{D}^{1 / 2}(\Omega)$. But the continuity of $A$ from $L^{2}(\Omega)$ to $L_{T}^{2} H_{D}^{1 / 2}(\Omega)$ is equivalent to the continuity of its adjoint

$$
\left(A^{\star} f\right)(t)=\int_{0}^{T} \mathrm{e}^{-i \tau \Delta_{D}} \chi f(\tau) \mathrm{d} \tau
$$

from $L_{T}^{2} H_{D}^{-1 / 2}(\Omega)$ to $L^{2}(\Omega)$, which in turn is equivalent to the continuity of $A A^{\star}$ from $L_{T}^{2} H_{D}^{-1 / 2}(\Omega)$ to $L_{T}^{2} H_{D}^{1 / 2}(\Omega)$. Write

$$
\begin{aligned}
\left(A A^{\star} f\right)(t) & =\int_{0}^{T} \chi \mathrm{e}^{i(t-\tau) \Delta_{D}} \chi f(\tau) \mathrm{d} \tau \\
& =\int_{0}^{t} \chi \mathrm{e}^{i(t-\tau) \Delta_{D}} \chi f(\tau) \mathrm{d} \tau+\int_{t}^{T} \chi \mathrm{e}^{i(t-\tau) \Delta_{D}} \chi f(\tau) \mathrm{d} \tau
\end{aligned}
$$

and it suffices to apply (2.14) with $s=-\frac{1}{2}$ (together with time inversion for the second term) in order to conclude that $A A^{\star}$ is bounded from $L_{T}^{2} H_{D}^{-1 / 2}(\Omega)$ to $L_{T}^{2} H_{D}^{1 / 2}(\Omega)$. This completes the proof of (2.15) for $s=0$.

We now prove (2.15) for $s=1$. Observe that the boundedness of $\chi \mathrm{e}^{i t \Delta_{D}}$ from $H_{D}^{1}(\Omega)$ to $L_{T}^{2} H_{D}^{3 / 2}(\Omega)$ is equivalent to the continuity of $\left(-\Delta_{D}+1\right) \chi \mathrm{e}^{i t \Delta_{D}}$ from $H_{D}^{1}(\Omega)$ to $L_{T}^{2} H_{D}^{-1 / 2}(\Omega)$. Write

$$
\left(-\Delta_{D}+1\right) \chi \mathrm{e}^{i t \Delta_{D}}=\chi\left(-\Delta_{D}+1\right) \mathrm{e}^{i t \Delta_{D}}-\left[\Delta_{D}, \chi\right] \mathrm{e}^{i t \Delta_{D}} .
$$


Let $\tilde{\chi} \in C_{0}^{\infty}\left(\mathbb{R}^{d}\right)$ be such that $\tilde{\chi}=1$ on the support of $\chi$. Then

$$
\begin{aligned}
\left\|\left[\Delta_{D}, \chi\right] \mathrm{e}^{i t \Delta_{D}} u_{0}\right\|_{L_{T}^{2} H_{D}^{-1 / 2}(\Omega)} & \lesssim\left\|\left[\Delta_{D}, \chi\right] \tilde{\chi} \mathrm{e}^{i t \Delta_{D}} u_{0}\right\|_{L_{T}^{2} H_{D}^{-1 / 2}(\Omega)} \\
& \lesssim\left\|\tilde{\chi} \mathrm{e}^{i t \Delta_{D}} u_{0}\right\|_{L_{T}^{2} H_{D}^{1 / 2}(\Omega)} \\
& \lesssim\left\|u_{0}\right\|_{L^{2}(\Omega)},
\end{aligned}
$$

where in the last line we used that (2.15) for $s=0$ is already established. Therefore $\left[\Delta_{D}, \chi\right] \mathrm{e}^{i t \Delta_{D}}$ is bounded from $L^{2}(\Omega)$ to $L_{T}^{2} H_{D}^{-1 / 2}(\Omega)$ and in particular from $H_{D}^{1}(\Omega)$ to $L_{T}^{2} H_{D}^{-1 / 2}(\Omega)$. Hence it remains to prove that the operator

$$
B:=\chi\left(-\Delta_{D}+1\right) \mathrm{e}^{i t \Delta_{D}}
$$

is bounded from $H_{D}^{1}(\Omega)$ to $L_{T}^{2} H_{D}^{-1 / 2}(\Omega)$ or equivalently that $B\left(-\Delta_{D}+1\right)^{-1} B^{\star}$ is bounded from $L_{T}^{2} H_{D}^{1 / 2}(\Omega)$ to $L_{T}^{2} H_{D}^{-1 / 2}(\Omega)$. An easy computation yields

$$
\begin{aligned}
\left(B\left(-\Delta_{D}+1\right)^{-1} B^{\star} f\right)(t) & =\int_{0}^{T} \chi\left(-\Delta_{D}+1\right) \mathrm{e}^{i(t-\tau) \Delta_{D}} \chi f(\tau) \mathrm{d} \tau \\
& =\left(-\Delta_{D}+1\right) \chi \int_{0}^{T} \mathrm{e}^{i(t-\tau) \Delta_{D}} \chi f(\tau) \mathrm{d} \tau+\left[\chi, \Delta_{D}\right] \int_{0}^{T} \mathrm{e}^{i(t-\tau) \Delta_{D}} \chi f(\tau) \mathrm{d} \tau .
\end{aligned}
$$

Observe that

$$
\left(-\Delta_{D}+1\right) \chi \int_{0}^{T} \mathrm{e}^{i(t-\tau) \Delta_{D}} \chi f(\tau) \mathrm{d} \tau=\left(-\Delta_{D}+1\right)\left(A A^{\star} f\right)(t)
$$

and therefore using (2.14) with $s=\frac{1}{2}$ together with a splitting of the integration on $[0, T]$ as shown above, we readily get that $\left(-\Delta_{D}+1\right) A A^{\star}$ is bounded from $L_{T}^{2} H_{D}^{1 / 2}(\Omega)$ to $L_{T}^{2} H_{D}^{-1 / 2}(\Omega)$. Next we write

$$
\left[\chi, \Delta_{D}\right] \int_{0}^{T} \mathrm{e}^{i(t-\tau) \Delta_{D}} \chi f(\tau) \mathrm{d} \tau=\left[\chi, \Delta_{D}\right]\left(A A^{\star} f\right)(t)
$$

and again due to (2.14) with $s=\frac{1}{2}$ we obtain the boundedness of $\left[\chi, \Delta_{D}\right] A A^{\star}$ from $L_{T}^{2} H_{D}^{1 / 2}(\Omega)$ to $L_{T}^{2} H_{D}^{-1 / 2}(\Omega)$. This completes the proof of (2.15) for $s=1$. We finally obtain (2.15) for $s \in[0,1]$ via an interpolation argument which ends the proof of Proposition 2.7.

Remark 2.9. If one considers the Neumann Laplacian $\Delta_{N}$, we can obtain a similar result as in Proposition 2.7, with constants depending on the time interval. Indeed take $\Psi \in C_{0}^{\infty}(\mathbb{R})$ equal to 1 close to 0 and decompose

$$
\begin{aligned}
u & =\Psi\left(-\Delta_{N}\right) u+(1-\Psi)\left(-\Delta_{N}\right) u, \\
f & =\Psi\left(-\Delta_{N}\right) f+(1-\Psi)\left(-\Delta_{N}\right) f, \\
v_{0} & =\Psi\left(-\Delta_{N}\right) v_{0}+(1-\Psi)\left(-\Delta_{N}\right) v_{0} .
\end{aligned}
$$

Taking into account Remark 2.3, we can apply the strategy of the proof of Proposition 2.7 to $(1-\Psi)\left(-\Delta_{N}\right) u$, $(1-\Psi)\left(-\Delta_{N}\right) f$ and $(1-\Psi)\left(-\Delta_{N}\right) v_{0}$ to obtain estimates similar as $(2.14),(2.15)$ for the contributions of these terms. To deal with the contributions of the other terms, we simply use the conservation of the $L^{2}$ norms and the fact that for these parts, the $L^{2}$ and $H_{N}^{k}$ norms are equivalent (due to the spectral cut-off). This argument gives an $L^{\infty}$ in time estimate for these terms which can be converted (using Hölder inequality) into an $L^{2}$ in time estimate. 


\subsection{Strichartz type estimates}

In the next proposition we show that away from the obstacle the free evolution satisfies the usual Strichartz bounds. We will use a strategy of [30] where similar considerations are performed in the context of $C^{2}$ short range perturbation of the free Laplacian on $\mathbb{R}^{d}$.

Proposition 2.10. For every $T>0$, for every $\chi \in C_{0}^{\infty}\left(\mathbb{R}^{d}\right), \chi=1$ close to $\Theta$ there exists $C>0$ such that

$$
\|(1-\chi) u\|_{L_{T}^{p} W^{s, q}(\Omega)} \leqslant C\left\|u_{0}\right\|_{H_{D}^{s}(\Omega)}
$$

where $s \in[0,1], u(t)=\mathrm{e}^{i t \Delta_{D}} u_{0}$ and $(p, q), p>2$, is any Strichartz admissible pair, i.e.

$$
\frac{2}{p}+\frac{d}{q}=\frac{d}{2}
$$

Proof. Set $v(t)=(1-\chi) \mathrm{e}^{i t \Delta_{D}} u_{0}$. Then $v$ satisfies the equation

$$
\left\{\begin{array}{l}
\left(i \partial_{t}+\Delta\right) v=\left[\Delta_{D},-\chi\right] u \\
v(0)=(1-\chi) u_{0}
\end{array}\right.
$$

Since $\chi=1$ close to $\Theta$, Eq. (2.19) can be regarded in the whole space $\mathbb{R}^{d}$. Hence

$$
v(t)=\mathrm{e}^{i t \Delta_{0}}(1-\chi) u_{0}+\int_{0}^{t} \mathrm{e}^{i(t-\tau) \Delta_{0}}\left[\Delta_{D},-\chi\right] u(\tau) \mathrm{d} \tau,
$$

where $\Delta_{0}$ is the free Laplacian on $\mathbb{R}^{d}$ and therefore the contribution of $(1-\chi) u_{0}$ satisfies the usual Strichartz estimate and we have reduced the problem to the study of

$$
w(t):=\int_{0}^{t} \mathrm{e}^{i(t-\tau) \Delta_{0}}\left[\Delta_{D},-\chi\right] u(\tau) \mathrm{d} \tau .
$$

Using Proposition 2.7, we get

$$
\left\|\left[\Delta_{D},-\chi\right] u\right\|_{L_{T}^{2} H^{-1 / 2}\left(\mathbb{R}^{d}\right)} \lesssim\left\|u_{0}\right\|_{L^{2}(\Omega)} .
$$

Let $\Lambda_{0} \varphi(t, x):=\mathrm{e}^{i t \Delta_{0}} \varphi(x)$. We proceed by using the smoothing effect for $\Lambda_{0}$. Applying inequality (1.10) in Corollary 2 and inequality (3.4) in Proposition 2 from [1], we have, for every cutoff function $\chi_{0}$ in $\mathbb{R}^{d}$,

$$
\left\|\left(1-\Delta_{0}\right)^{1 / 4}\left(\chi_{0} \Lambda_{0} \varphi\right)\right\|_{L^{2}\left([0, T] \times \mathbb{R}^{d}\right)} \lesssim\|\varphi\|_{L^{2}\left(\mathbb{R}^{d}\right)} .
$$

The dual inequality reads

$$
\left\|\Lambda_{0}^{*}\left(\chi_{0}\left(1-\Delta_{0}\right)^{1 / 4} \psi\right)\right\|_{L^{2}\left(\mathbb{R}^{d}\right)} \lesssim\|\psi\|_{L^{2}\left([0, T] \times \mathbb{R}^{d}\right)} .
$$

Combining with Strichartz estimates on $\mathbb{R}^{d}$ for $\Lambda_{0}$, this yields

$$
\left\|\Lambda_{0} \Lambda_{0}^{*}\left(\chi_{0}\left(1-\Delta_{0}\right)^{1 / 4} \psi\right)\right\|_{L_{T}^{p} L^{q}\left(\mathbb{R}^{d}\right)} \lesssim\left\|u_{0}\right\|_{L^{2}(\Omega)}
$$

Notice that

$$
\Lambda_{0} \Lambda_{0}^{*}(f)(t)=\int_{0}^{T} \mathrm{e}^{i(t-\tau) \Delta_{0}} f(\tau) \mathrm{d} \tau
$$


However we are interested in estimating $w(t)$ defined by (2.20) rather than

$$
\Lambda_{0} \Lambda_{0}^{*}\left(\left[\Delta_{D},-\chi\right] u\right) \text {. }
$$

For this it suffices to use the following result due to Christ and Kiselev [16].

Theorem (M. Christ and A. Kiselev). Consider a bounded operator

$$
T: L^{p}\left(\mathbb{R} ; B_{1}\right) \rightarrow L^{q}\left(\mathbb{R} ; B_{2}\right)
$$

given by a locally integrable kernel $K(t, s)$ with values in bounded operators from $B_{1}$ to $B_{2}$ where $B_{1}$ and $B_{2}$ are Banach spaces. Suppose that $p<q$. Then the operator

$$
\widetilde{T} \psi(t)=\int_{s<t} K(t, s) \psi(s) \mathrm{d} s
$$

is bounded from $L^{p}\left(\mathbb{R} ; B_{1}\right)$ to $L^{q}\left(\mathbb{R} ; B_{2}\right)$ and

$$
\|\widetilde{T}\|_{L^{p}\left(\mathbb{R} ; B_{1}\right) \rightarrow L^{q}\left(\mathbb{R} ; B_{2}\right)} \lesssim\left(1-2^{-\left(p^{-1}-q^{-1}\right)}\right)^{-1}\|T\|_{L^{p}\left(\mathbb{R} ; B_{1}\right) \rightarrow L^{q}\left(\mathbb{R} ; B_{2}\right)}
$$

In view of (2.21), we apply Christ-Kiselev's theorem to

$$
K(t, s)=\mathbf{1}_{[0, T]}(t) \mathbf{1}_{[0, T]}(s) \mathrm{e}^{i(t-s) \Delta_{0}} \chi_{0}\left(1-\Delta_{0}\right)^{1 / 4}
$$

and we set $\psi=\left(1-\Delta_{0}\right)^{-1 / 4}\left[\Delta_{D},-\chi\right] u$ with $\chi_{0}=1$ near the support of $\chi$. This yields, for $p>2$,

$$
\|w\|_{L_{T}^{p} L^{q}\left(\mathbb{R}^{d}\right)} \lesssim\left\|\left[\Delta_{D},-\chi\right] u\right\|_{L_{T}^{2} H^{-1 / 2}\left(\mathbb{R}^{d}\right)} \lesssim\left\|u_{0}\right\|_{L^{2}(\Omega)}
$$

This completes the proof for $s=0$.

The case $s=1$ can be treated similarly simply by differentiating the first equation of (2.19), considered as equation on the whole space $\mathbb{R}^{d}$. Since we established (2.17) for $s=0$ and $s=1$ an interpolation argument completes the proof of Proposition 2.10.

Now we state a Strichartz estimate (with loss of derivative) for $\mathrm{e}^{i t \Delta_{D}}$.

Proposition 2.11. For every $T>0$ there exists $C>0$ such that

$$
\|u\|_{L_{T}^{p} W^{s, q}(\Omega)} \leqslant C\left\|u_{0}\right\|_{H_{D}^{s+1 / p}(\Omega)},
$$

where $s \in[0,1], u(t)=\mathrm{e}^{i t \Delta_{D}} u_{0}$ and $(p, q), p>2$, satisfies $(2.18)$.

Remark 2.12. In [9], Strichartz inequalities as (2.22) are proven for the free Schrödinger equation posed on a compact Riemannian manifold (without boundary). Although the estimates are the same, the ideas behind are very different. In [9], the loss of derivatives (optimal for the endpoint cases on the sphere) came from the fact that we were able to prove the usual estimates (without loss) only for small time intervals (depending on the frequency). Here the loss (certainly not optimal ... ) comes from the fact that close to the boundary, we perform simply Sobolev embeddings together with the local smoothing. The gain arising from the smoothing effect tells us that the wave spends few time close to the obstacle.

Remark 2.13. In [29] Smith and Sogge prove that the wave equation posed on the exterior of strictly convex obstacle satisfies the same Strichartz estimates as the solution of the wave equation posed on $\mathbb{R}^{d}$. It is natural to expect that the techniques of [29] combined with the semi-classical approach of [9] can provide the full set of Strichartz inequalities, at least locally in time, for the Schrödinger equation posed on the exterior of strictly convex 
obstacle. Such a result would extend the well-posedness theory of the flat space to the case of the exterior of a strictly convex obstacle.

Proof of Proposition 2.11. Consider $\chi \in C_{0}^{\infty}\left(\mathbb{R}^{d}\right)$ equal to 1 close to $\Theta$ and decompose

$$
u(t)=\chi \mathrm{e}^{i t \Delta_{D}} u_{0}+(1-\chi) \mathrm{e}^{i t \Delta_{D}} u_{0}:=v(t)+w(t) .
$$

Due to Proposition 2.10, we obtain that $w(t)$ satisfies the usual Strichartz estimates (without losses) and therefore we only need to evaluate $v(t)$. Using Proposition 2.7, we get

$$
\|v\|_{L_{T}^{2} H_{D}^{1}(\Omega)} \lesssim\left\|u_{0}\right\|_{H_{D}^{1 / 2}(\Omega)} .
$$

Next we use an energy argument to deduce,

$$
\|v\|_{L_{T}^{\infty} L^{2}(\Omega)} \lesssim\left\|u_{0}\right\|_{L^{2}(\Omega)} .
$$

Interpolating between (2.23) and (2.24) with weights $\frac{2}{p}$ and $1-\frac{2}{p}$ respectively gives

$$
\|v\|_{L_{T}^{p} H_{D}^{2 / p}(\Omega)} \lesssim\left\|u_{0}\right\|_{H_{D}^{1 / p}(\Omega)} \cdot
$$

Since $\frac{2}{p}+\frac{d}{q}=\frac{d}{2}$, using Proposition 2.1 we have that $H_{D}^{2 / p}(\Omega) \subset L^{q}(\Omega)$ and the embedding is continuous, therefore

$$
\|v\|_{L_{T}^{p} L^{q}(\Omega)} \lesssim\left\|u_{0}\right\|_{H_{D}^{1 / p}(\Omega)}
$$

which completes the proof of Proposition 2.11 when $s=0$.

Next we consider the case $s=1$. Applying an energy argument, we get

$$
\|v\|_{L_{T}^{\infty} H_{D}^{p /(p-2)}(\Omega)} \lesssim\left\|u_{0}\right\|_{H_{D}^{p /(p-2)}(\Omega)} .
$$

Interpolation between (2.23) and (2.25) with weights $\frac{2}{p}$ and $1-\frac{2}{p}$ respectively gives

$$
\|v\|_{L_{T}^{p} H_{D}^{1+2 / p}(\Omega)} \lesssim\left\|u_{0}\right\|_{H_{D}^{1+1 / p}(\Omega)} .
$$

Due to Proposition 2.1 the continuous embedding $H_{D}^{1+2 / p}(\Omega) \subset W^{1, q}(\Omega)$ holds which together with (2.26) ends the proof for $s=1$. The case $s \in[0,1]$ can now be treated by interpolation.

Now we state the main result of this section.

Proposition 2.14. For every $T \in] 0,1]$ there exists $C>0$ such that

$$
\|u\|_{L_{T}^{p} W^{s, q}(\Omega)} \leqslant C\left\|u_{0}\right\|_{H_{D}^{s}(\Omega)},
$$

where $s \in[0,1], u(t)=\mathrm{e}^{i t \Delta_{D}} u_{0}$ and $(p, q), p \geqslant 2$ satisfies

$$
\frac{1}{p}+\frac{d}{q}=\frac{d}{2}
$$

Moreover

$$
\|u\|_{L_{T}^{p} W^{s, q}(\Omega)} \leqslant C\|f\|_{L_{T}^{1} H_{D}^{s}(\Omega)},
$$

where $s \in[0,1], u(t)=\int_{0}^{t} \mathrm{e}^{i(t-\tau) \Delta_{D}} f(\tau) \mathrm{d} \tau$ and $(p, q), p>2$ satisfies (2.28).

Proof. Let $\chi \in C_{0}^{\infty}\left(\mathbb{R}^{d}\right)$ such that $\chi=1$ close to $\Theta$. The triangle inequality yields,

$$
\|u\|_{L_{T}^{p} W^{s, q}(\Omega)} \leqslant\|\chi u\|_{L_{T}^{p} W^{s, q}(\Omega)}+\|(1-\chi) u\|_{L_{T}^{p} W^{s, q}(\Omega)}
$$


and hence it is sufficient to evaluate each term in the right-hand side of the above inequality. Using Proposition 2.7, we obtain

$$
\|\chi u\|_{L_{T}^{2} H_{D}^{s+1 / 2}(\Omega)} \lesssim\left\|u_{0}\right\|_{H_{D}^{s}(\Omega)} .
$$

Next an energy argument yields,

$$
\|\chi u\|_{L_{T}^{\infty} H_{D}^{s}(\Omega)} \lesssim\left\|u_{0}\right\|_{H_{D}^{s}(\Omega)} .
$$

Interpolation between (2.30) and (2.31) with weights $\frac{2}{p}$ and $1-\frac{2}{p}$ respectively yields,

$$
\|\chi u\|_{L_{T}^{p} H_{D}^{s+1 / p}(\Omega)} \lesssim\left\|u_{0}\right\|_{H_{D}^{s}(\Omega)} .
$$

Next using the embedding $H_{D}^{s+1 / p}(\Omega) \subset W^{s, q}(\Omega), s \in[0,1], p>2$, where the pair $(p, q)$ satisfies (2.28) (see Proposition 2.1), we obtain,

$$
\|\chi u\|_{L_{T}^{p} W^{s, q}(\Omega)} \lesssim\left\|u_{0}\right\|_{H_{D}^{s}(\Omega)}
$$

We next bound $(1-\chi) u$. Fix $(p, q), p>2$ satisfying (2.28). Let $p^{\star}$ be such that $\frac{2}{p^{\star}}+\frac{d}{q}=\frac{d}{2}$, i.e., $p^{\star}=2 p$. Using Hölder's inequality in time and Proposition 2.10, we get

$$
\|(1-\chi) u\|_{L_{T}^{p} W^{s, q}(\Omega)} \lesssim T^{1 /(2 p)}\|(1-\chi) u\|_{L_{T}^{p^{\star} W^{s, q}(\Omega)}} \lesssim\left\|u_{0}\right\|_{H_{D}^{s}(\Omega)} .
$$

This completes the proof of (2.27). Estimate (2.29) follows from (2.27) and the Minkowski integral inequality applied in time variable.

The next proposition is a consequence of (2.27) and Christ-Kiselev's theorem.

Proposition 2.15. For every $T \in] 0,1]$ there exists $C>0$ such that

$$
\|u\|_{L_{T}^{p} W^{s, q}(\Omega)} \leqslant C\left\|\left(1-\Delta_{D}\right)^{s / 2} f\right\|_{L_{T}^{\tilde{p}} L^{\tilde{q}}(\Omega)}, \quad s \in[0,1],
$$

where $u(t)=\int_{0}^{t} \mathrm{e}^{i(t-\tau) \Delta_{D}} f(\tau) \mathrm{d} \tau,(p, q), p>2$, satisfies (2.28) and $(\tilde{p}, \tilde{q}), \tilde{p} \in[1,2[$, satisfies

$$
\frac{1}{\tilde{p}}+\frac{d}{\tilde{q}}=1+\frac{d}{2}
$$

i.e. $(\tilde{p} /(\tilde{p}-1), \tilde{q} /(\tilde{q}-1))$ satisfies (2.28).

Remark 2.16. Notice that in estimate (2.32) the pairs $(p, q)$ and $(\tilde{p}, \tilde{q})$ are not necessarily conjugate Hölder exponents.

Proof of Proposition 2.15. Due to Christ-Kiselev's theorem, it is sufficient to evaluate

$$
w(t):=\int_{0}^{T} \mathrm{e}^{i(t-\tau) \Delta_{D}} f(\tau) \mathrm{d} \tau .
$$

Using (2.27), we obtain

$$
\|w\|_{L_{T}^{p} W^{s, q}(\Omega)} \lesssim\|F\|_{H_{D}^{s}(\Omega)}, \quad s \in[0,1],
$$

where $F=\int_{0}^{T} \mathrm{e}^{-i \tau \Delta_{D}} f(\tau) \mathrm{d} \tau$. Next the dual of (2.27) gives,

$$
\|F\|_{H_{D}^{s}(\Omega)} \lesssim\left\|\left(1-\Delta_{D}\right)^{s / 2} f\right\|_{L_{T}^{\tilde{p}} L^{\tilde{q}}(\Omega)}, \quad s \in[0,1],
$$

where $(\tilde{p}, \tilde{q}), \tilde{p} \in[1,2[$, satisfies (2.33). This completes the proof of Proposition 2.15. 


\section{Proof of Theorem 1}

We prove Theorem 1 for positive times. Similar arguments can be performed for negative times. The initial boundary value problem (1.1)-(1.2)-(1.3) can be written as an integral equation (Duhamel form),

$$
u(t)=\mathrm{e}^{i t \Delta_{D}} u_{0}+\int_{0}^{t} \mathrm{e}^{i(t-\tau) \Delta_{D}} F(u(\tau)) \mathrm{d} \tau,
$$

where the nonlinearity $F$ is as described in the introduction. The assumptions on $F$ and on the potential $V$ imply the following pointwise estimates,

$$
\begin{aligned}
& |F(u)| \lesssim|u|\left(1+|u|^{\alpha}\right), \\
& |\nabla F(u)| \lesssim|\nabla u|\left(1+|u|^{\alpha}\right)
\end{aligned}
$$

and moreover by writing

$$
F(u)-F(v)=\int_{0}^{1} F^{\prime}(t u+(1-t) v)(u-v) \mathrm{d} t
$$

we obtain

$$
\begin{aligned}
& |F(u)-F(v)| \lesssim|u-v|\left(1+|u|^{\alpha}+|v|^{\alpha}\right), \\
& |\nabla(F(u)-F(v))| \lesssim|\nabla(u-v)|\left(1+|u|^{\alpha}+|v|^{\alpha}\right)+|u-v|(|\nabla u|+|\nabla v|)(1+|u|+|v|)^{\max \{\alpha-1,0\}} .
\end{aligned}
$$

We note that assumptions (3.2)-(3.5) on the nonlinear interaction would be sufficient for the local well-posedness analysis.

\subsection{Uniqueness}

As a first consequence of our linear estimates, we prove the uniqueness. Let $u, v$ with $u(0)=v(0)$ be two solutions both in $C\left([0, T] ; H_{0}^{1}(\Omega)\right)$. By the Sobolev embedding $u, v \in L_{T}^{\infty} L^{2 d /(d-2)}(\Omega)$ hence $F(u), F(v)$ are in the sum of $L_{T}^{\infty} L^{2 d /((d-2)(\alpha+1))}(\Omega)$ and $L_{T}^{\infty} L^{2}(\Omega)$. The condition $\alpha<\frac{2}{d-2}$ implies that $2 d /((d-2)(1+\alpha))$ is bigger than $2 d /(d+1)$ which is the end point value for $\tilde{q}$ in (2.32). As a consequence $u$ and $v$ are both in $L_{T}^{p} L^{q}(\Omega)$ for any pair $(p, q), p>2$, satisfying (2.28). Therefore the uniqueness claim of Theorem 1 will be a consequence of the next proposition.

Proposition 3.1. Let $u$ and $v$ be two solutions of (1.1)-(1.2)-(1.3) lying in $C\left([0, T] ; H_{0}^{1}(\Omega)\right)$. Then there exist $\beta \in\left[\alpha, \frac{2}{d-2}[, p>2\right.$, and $\theta>0$ such that for $T \in] 0,1[$,

$$
\|u-v\|_{Y_{T}} \lesssim\|u(0)-v(0)\|_{L^{2}(\Omega)}+T^{\theta}\left(1+\|u\|_{L_{T}^{\infty} H_{0}^{1}(\Omega)}^{\beta}+\|v\|_{L_{T}^{\infty} H_{0}^{1}(\Omega)}^{\beta}\right)\|u-v\|_{Y_{T}},
$$

where $Y_{T}=L_{T}^{\infty} L^{2}(\Omega) \cap L_{T}^{p} L^{q}(\Omega), \frac{1}{p}+\frac{d}{q}=\frac{d}{2}$, is equipped with the natural norm.

Proof. Let $\psi \in C_{0}^{\infty}(\mathbb{R})$ be such that $\psi(x)=0$ for $|x|<1$ and $\psi(x)=1$ for $|x|>2$. By the splitting

$$
F(u)-F(v)=\psi\left(|u|^{2}+|v|^{2}\right)(F(u)-F(v))+\left(1-\psi\left(|u|^{2}+|v|^{2}\right)\right)(F(u)-F(v))
$$

and Propositions 2.14 and 2.15, we infer the bound

$$
\|u-v\|_{Y_{T}} \lesssim\|u(0)-v(0)\|_{L^{2}}+T\|u-v\|_{L_{T}^{\infty} L^{2}}+\left\|\left(1-\psi\left(|u|^{2}+|v|^{2}\right)\right)(F(u)-F(v))\right\|_{L_{T}^{\tilde{p}} L^{\tilde{q}}},
$$


where $(\tilde{p}, \tilde{q}), \tilde{p} \in\left[1,2\left[\right.\right.$, satisfies (2.33). We choose $\tilde{p}$ such that $\frac{1}{\tilde{p}}=\frac{1}{2}+\delta$, where $\delta>0$ is small enough to be chosen later. We further take the parameter $p$ involved in the statement as $\frac{1}{p}=\frac{1}{2}-\delta$. Next, using the Hölder inequality and the Sobolev embedding, we get

$$
\begin{aligned}
\left\|\left(1-\psi\left(|u|^{2}+|v|^{2}\right)\right)(F(u)-F(v))\right\|_{L_{T}^{\tilde{p}} L^{\tilde{q}}} & \lesssim\|u-v\|_{L_{T}^{2} L^{q}}\left(\|u\|_{L_{T}^{\beta p_{1}} L^{\beta q_{1}}}^{\beta}+\|v\|_{L_{T}^{\beta p_{1}} L^{\beta q_{1}}}^{\beta}\right) \\
& \lesssim T^{1 / 2-1 / p}\|u-v\|_{L_{T}^{p} L^{q}}\left(\|u\|_{L_{T}^{\infty} H_{0}^{1}}^{\beta}+\|v\|_{L_{T}^{\infty} H_{0}^{1}}^{\beta},\right.
\end{aligned}
$$

where $\beta \in\left[\alpha, \frac{2}{d-2}\right.$ [ is to be chosen later and the parameters $p_{1}, q_{1}$ satisfy the Hölder inequality conditions $\frac{1}{\tilde{p}}=\frac{1}{2}+\frac{1}{p_{1}}$ and $\frac{1}{\tilde{q}}=\frac{1}{q}+\frac{1}{q_{1}}$, i.e., $p_{1}=\delta^{-1}$ and $\frac{1}{q_{1}}=\frac{1-2 \delta}{d}$. Finally the Sobolev inequality condition $2<\beta q_{1}<\frac{2 d}{d-2}$ can be written as

$$
\frac{2}{d}-\frac{4 \delta}{d}<\beta<\frac{2}{d-2}-\frac{4 \delta}{d-2}
$$

We can ensure (3.6) by choosing $\delta$ small enough and $\beta$ close enough to $\frac{2}{d-2}$. This completes the proof of Proposition 3.1.

\subsection{Proof of Theorem 1 in $2 d$}

(See also [9, Proposition 3.1].) In this subsection we perform the proof of Theorem 1 for $d=2$. We shall only make use of Proposition 2.11. Consider a plane domain $\Omega$ which is the complementary of a compact smooth nontrapping obstacle $\Theta$. Fix a pair $(p, q) \in \mathbb{R}^{2}$ such that $p>\alpha$ and $\frac{1}{p}+\frac{1}{q}=\frac{1}{2}$. The aim is to show that for sufficiently small $T>0$ we can solve (3.1) by a Picard iteration scheme in the space

$$
X_{T}:=L_{T}^{\infty} H_{0}^{1}(\Omega) \cap L_{T}^{p} W^{1-1 / p, q}(\Omega),
$$

equipped with the natural norm

$$
\|u\|_{X_{T}}:=\|u\|_{L_{T}^{\infty} H_{0}^{1}(\Omega)}+\|u\|_{L_{T}^{p} W^{1-1 / p, q}(\Omega)} .
$$

Proposition 2.11 gives that the free evolution $\mathrm{e}^{i t \Delta_{D}}$ is bounded from $H_{0}^{1}(\Omega)$ to $X_{T}$. Next we define a map $\Lambda$ as follows

$$
(\Lambda f)(t):=\int_{0}^{t} \mathrm{e}^{i(t-\tau) \Delta_{D}} f(\tau) \mathrm{d} \tau .
$$

We claim that the map $\Lambda$ is bounded from $L_{T}^{1} H_{0}^{1}(\Omega)$ to $X_{T}$, i.e., the following estimate holds,

$$
\|\Lambda f\|_{X_{T}} \lesssim\|f\|_{L_{T}^{1} H_{0}^{1}(\Omega)} .
$$

Indeed, the boundedness of $\Lambda$ from $L_{T}^{1} H_{0}^{1}(\Omega)$ to $L_{T}^{\infty} H_{0}^{1}(\Omega)$ follows from an energy argument while the boundedness from $L_{T}^{1} H_{0}^{1}(\Omega)$ to $L_{T}^{p} W^{1-1 / p, q}(\Omega)$ results from Proposition 2.11 and the Minkowski integral inequality. This proves (3.7).

Next we bound the nonlinear term $F(u)$ in the space $L_{T}^{1} H_{0}^{1}(\Omega)$. Using (3.2), (3.3) and the embedding $H_{0}^{1}(\Omega) \subset L^{2(\alpha+1)}(\Omega)$ (see Proposition 2.1), we obtain

$$
\|F(u)\|_{L_{T}^{1} H_{0}^{1}(\Omega)} \lesssim\|u\|_{L_{T}^{\infty} H_{0}^{1}(\Omega)}\left(T+\|u\|_{L_{T}^{\alpha} L^{\infty}(\Omega)}^{\alpha}\right)+T\|u\|_{L_{T}^{\infty} H_{0}^{1}(\Omega)}^{\alpha+1} .
$$

Due to the assumption $p>\alpha$, a use of Hölder inequality in time yields,

$$
\|u\|_{L_{T}^{\alpha} L^{\infty}(\Omega)}^{\alpha} \lesssim T^{\theta}\|u\|_{L_{T}^{p} L^{\infty}(\Omega)}^{\alpha},
$$


where $\theta=1-\frac{\alpha}{p}$. Next since $q>2$ we have that $1-\frac{1}{p}>\frac{2}{q}$ and therefore due to the continuous embedding $W^{1-1 / p, q}(\Omega) \subset L^{\infty}(\Omega)$ (see Proposition 2.1), we get

$$
\|u\|_{L_{T}^{\alpha} L^{\infty}(\Omega)}^{\alpha} \lesssim T^{\theta}\|u\|_{L_{T}^{p} W^{1-1 / p, q}(\Omega)}^{\alpha} \lesssim T^{\theta}\|u\|_{X_{T}}^{\alpha} .
$$

Plugging the last estimate into (3.8) we deduce that for every $\alpha$ there exists $\theta_{1}>0$ such that for $0 \leqslant T \leqslant 1$,

$$
\|F(u)\|_{L_{T}^{1} H_{0}^{1}(\Omega)} \lesssim T^{\theta_{1}}\|u\|_{X_{T}}\left(1+\|u\|_{X_{T}}^{\alpha}\right) .
$$

Similarly to the proof of (3.9), we can show that for every $\alpha$ there exists $\theta_{2}>0$ such that for $0 \leqslant T \leqslant 1$,

$$
\|F(u)-F(v)\|_{L_{T}^{1} H_{0}^{1}(\Omega)} \lesssim T^{\theta_{2}}\|u-v\|_{X_{T}}\left(1+\|u\|_{X_{T}}^{\alpha}+\|v\|_{X_{T}}^{\alpha}\right) .
$$

It is now a standard issue to see that (3.9), (3.10) together with (3.7) allow us to solve (3.1) via Picard iteration scheme in $X_{T}$ provided $T$ be sufficiently small. This yields the local well-posedness and the Lipschitz property of the flow map. For the global well-posedness we first observe that the smallness assumption on $T$ in the local well-posedness depends only on the size of $H_{0}^{1}(\Omega)$ norm of $u_{0}$ and not on its profile. The rigorous derivation of the conservation laws (1.4a), (1.4b) can be done by a standard approximation argument (see [13], Section 4). Next due to (1.4a), (1.4b), the assumption on $V$ and Gagliardo-Nirenberg inequality we obtain the control on the $H_{0}^{1}(\Omega)$ norm of the local solutions solution (see [13], Section 6). Therefore we can reiterate the local well-posedness argument and extend the local solution to an arbitrary time interval.

\subsection{Proof of Theorem 1 in three space dimensions}

For $T>0$, we define a Banach space

$$
X_{T}:=L_{T}^{\infty} H_{0}^{1}(\Omega) \cap L_{T}^{3} W^{1,18 / 7}(\Omega),
$$

equipped with the norm

$$
\|u\|_{X_{T}}:=\|u\|_{L_{T}^{\infty} H_{0}^{1}(\Omega)}+\|u\|_{L_{T}^{3} W^{1,18 / 7}(\Omega)} .
$$

The next proposition is a direct consequence of Proposition 2.14 .

Proposition 3.2. Define a nonlinear map $\Phi$ as follows,

$$
(\Phi(u))(t):=\int_{0}^{t} \mathrm{e}^{i(t-\tau) \Delta_{D}} F(u(\tau)) \mathrm{d} \tau .
$$

Then

$$
\begin{aligned}
& \|\Phi(u)\|_{X_{T}} \lesssim\|F(u)\|_{L_{T}^{1} H_{0}^{1}(\Omega)}, \\
& \|\Phi(u)-\Phi(v)\|_{X_{T}} \lesssim\|F(u)-F(v)\|_{L_{T}^{1} H_{0}^{1}(\Omega)} .
\end{aligned}
$$

The next proposition contains the nonlinear estimate involved in the proof of Theorem 1 in $3 d$.

Proposition 3.3. For every $\alpha \in] 0,2]$ there exists $\theta_{1}(\alpha)>0$ and $\theta_{2}(\alpha) \geqslant 0$ such that for $\left.\left.T \in\right] 0,1\right]$,

$$
\begin{aligned}
& \|F(u)\|_{L_{T}^{1} H_{0}^{1}(\Omega)} \lesssim T^{\theta_{1}(\alpha)}\|u\|_{X_{T}}+T^{\theta_{2}(\alpha)}\|u\|_{X_{T}}^{3}, \\
& \|F(u)-F(v)\|_{L_{T}^{1} H_{0}^{1}(\Omega)} \lesssim\|u-v\|_{X_{T}}\left(T^{\theta_{1}(\alpha)}+T^{\theta_{2}(\alpha)}\left(1+\|u\|_{X_{T}}^{2}+\|v\|_{X_{T}}^{2}\right)\right),
\end{aligned}
$$

where $\theta_{2}(\alpha)=0$ only for $\alpha=2$. 
Proof. Using the embedding $\left.\left.H_{0}^{1}(\Omega) \subset L^{2(\alpha+1)}(\Omega), \alpha \in\right] 0,2\right]$ (see Proposition 2.1), we obtain

$$
\begin{aligned}
\|F(u)\|_{L_{T}^{1} L^{2}(\Omega)} & \lesssim\|u\|_{L_{T}^{1} H_{0}^{1}(\Omega)}\left(1+\|u\|_{L_{T}^{1} H_{0}^{1}(\Omega)}^{\alpha}\right) \\
& \lesssim T\|u\|_{X_{T}}\left(1+\|u\|_{X_{T}}^{2}\right) .
\end{aligned}
$$

Using (3.3) we obtain that

$$
|\nabla F(u)| \lesssim|\nabla u|\left(1+|u|^{\beta}\right),
$$

where $\alpha \leqslant \beta \leqslant 2$. Since

$$
\|\nabla u\|_{L_{T}^{1} L^{2}(\Omega)} \lesssim T\|u\|_{X_{T}}
$$

we need to bound $|u|^{\beta} \nabla u$ in $L_{T}^{1} L^{2}(\Omega)$ for a suitable $\beta$ (the auxiliary parameter $\beta$ will be chosen close enough to 2). We now claim that

$$
\|u\|_{L_{T}^{p} W^{1, q}(\Omega)} \lesssim\|u\|_{X_{T}}, \quad \frac{1}{3 p}+\frac{1}{q}=\frac{1}{2}, p \geqslant 3 .
$$

Estimate (3.16) follows clearly from the definition of $X_{T}$. Next using Hölder inequality we get for $\beta>1$,

$$
\left\||u|^{\beta-1} u \nabla u\right\|_{L_{T}^{1} L^{2}(\Omega)} \lesssim\left\||u|^{\beta-1}\right\|_{L_{T}^{3} L^{18}(\Omega)}\|u\|_{L_{T}^{3} L^{18}(\Omega)}\|\nabla u\|_{L_{T}^{3} L^{18 / 7}(\Omega)}
$$

and moreover using (3.16) and the Sobolev embedding $W^{1,18 / 7}(\Omega) \subset L^{18}(\Omega)$ (see Proposition 2.1), we deduce that

$$
\left\||u|^{\beta-1} u \nabla u\right\|_{L_{T}^{1} L^{2}(\Omega)} \lesssim\|u\|_{L_{T}^{3(\beta-1)} L^{18(\beta-1)}(\Omega)}^{\beta-1}\|u\|_{X_{T}}^{2} .
$$

Choose $q$ such that $\frac{1}{q}-\frac{1}{18(\beta-1)}=\frac{1}{3}$. It is always possible to have $q \geqslant 2$ by choosing $\beta$ close enough to 2 . Next using Proposition 2.1, we obtain

$$
\|u\|_{L_{T}^{3(\beta-1)} L^{18(\beta-1)}(\Omega)} \lesssim\|u\|_{L_{T}^{3(\beta-1)} W^{1, q}(\Omega)} .
$$

Chose now $p$ such that $\frac{1}{3 p}+\frac{1}{q}=\frac{1}{2}$. Since $\beta \leqslant 2$, we have that $q \leqslant \frac{18}{7}$ and therefore $p \geqslant 3$. Moreover since $\beta \leqslant 2$ we have that $\frac{1}{p} \leqslant \frac{1}{3(\beta-1)}$ and an easy computation yields $\frac{1}{3(\beta-1)}-\frac{1}{p}=\frac{2-\beta}{2(\beta-1)}$. Hence we obtain that for $1<\beta \leqslant 2$,

$$
\|u\|_{L_{T}^{3(\beta-1)} W^{1, q}(\Omega)} \lesssim T^{(2-\beta) /(2(\beta-1))}\|u\|_{X_{T}}
$$

and therefore (see (3.17)),

$$
\left\||u|^{\beta} \nabla u\right\|_{L_{T}^{1} H_{0}^{1}(\Omega)} \lesssim T^{(2-\beta) /(2(\beta-1))}\|u\|_{X_{T}}^{\beta+1}
$$

which in turn together with (3.14) and (3.15) gives

$$
\begin{aligned}
\|F(u)\|_{L_{T}^{1} H_{0}^{1}(\Omega)} & \lesssim T\|u\|_{X_{T}}\left(1+\|u\|_{X_{T}}^{2}\right)+T^{(2-\beta) /(2(\beta-1))}\|u\|_{X_{T}}^{\beta+1} \\
& \lesssim T^{\theta_{1}(\alpha)}\|u\|_{X_{T}}+T^{\theta_{2}(\alpha)}\|u\|_{X_{T}}^{3},
\end{aligned}
$$

where $\theta_{2}(\alpha)=\min \left\{1, \frac{2-\beta}{2(\beta-1)}\right\}$ and

$$
\theta_{1}(\alpha)= \begin{cases}1, & \text { if } \alpha=2, \\ \theta_{2}(\alpha), & \text { if } \alpha<2 .\end{cases}
$$

This ends the proof of (3.12). The proof of (3.13) is similar by invoking (3.4), (3.5). The only new feature in the analysis is the estimate of the quadratic expression $|u-v|(|\nabla u|+|\nabla v|)$. Thus we need to bound say $(u-v) \nabla u$ in $L_{T}^{1} L^{2}(\Omega)$. This can be done by using Hölder inequality, Proposition 2.1 and (3.16) as follows, 


$$
\begin{aligned}
\|(u-v) \nabla u\|_{L_{T}^{1} L^{2}(\Omega)} & \lesssim\|u-v\|_{L_{T}^{3 / 2} L^{9}(\Omega)}\|\nabla u\|_{L_{T}^{3} L^{18 / 7}(\Omega)} \lesssim\|u-v\|_{L_{T}^{3 / 2} W^{1,9 / 4}(\Omega)}\|u\|_{X_{T}} \\
& \lesssim T^{1 / 2}\|u-v\|_{L_{T}^{6} W^{1,9 / 4}(\Omega)}\|u\|_{X_{T}} \lesssim T^{1 / 2}\|u-v\|_{X_{T}}\|u\|_{X_{T}} .
\end{aligned}
$$

This completes the proof of Proposition 3.3.

Using Propositions 3.2 and 3.3, we obtain that for $\alpha \in] 0,2[$ there exists $\theta(\alpha)>0$ such that for $T \in] 0,1]$,

$$
\|\Phi(u)\|_{X_{T}} \lesssim T^{\theta(\alpha)}\|u\|_{X_{T}}\left(1+\|u\|_{X_{T}}^{2}\right)
$$

and

$$
\|\Phi(u)-\Phi(v)\|_{X_{T}} \lesssim T^{\theta(\alpha)}\|u-v\|_{X_{T}}\left(1+\|u\|_{X_{T}}^{2}+\|v\|_{X_{T}}^{2}\right) .
$$

Since $\mathrm{e}^{i t \Delta_{D}}$ is an isometry on $H_{0}^{1}(\Omega)$ we deduce that for $\left.\alpha \in\right] 0,2[$ the map

$$
\left(K_{u_{0}}(u)\right)(t):=\mathrm{e}^{i t \Delta_{D}} u_{0}+(\Phi(u))(t)
$$

is a contraction in a suitable ball of $X_{T}$, provided $T$ be sufficiently small. Therefore if we consider the sequence $\left\{v_{n}\right\}_{n=0}^{\infty}, v_{n} \in X_{T}$ such that $v_{0}=0, v_{n+1}=K_{u_{0}}\left(v_{n}\right)$ then $v_{n}$ converges in $X_{T}$ to the unique solution in $X_{T}$ of the integral equation

$$
u(t)=\mathrm{e}^{i t \Delta_{D}} u_{0}+\int_{0}^{t} \mathrm{e}^{i(t-\tau) \Delta_{D}} F(u(\tau)) \mathrm{d} \tau
$$

which implies the local well-posedness for $\alpha<2$. If $\alpha=2$, again using Propositions 3.2 and 3.3, we obtain that $K_{u_{0}}$ is a contraction on a suitable ball of $X_{T}$ only if in addition we impose a smallness assumption on $\left\|u_{0}\right\|_{H_{0}^{1}(\Omega)}$. The global well-posedness can be obtained as explained in the previous section. The Lipschitz continuity of the flow map on bounded sets of $H_{0}^{1}(\Omega)$ for small time intervals is a consequence of (3.19) while for an arbitrary time the argument should be iterated using the control on $H_{0}^{1}(\Omega)$ norm provided by the energy conservation.

\subsection{Higher dimensions}

In dimension $d \geqslant 4$, we deal with the space

$$
X_{T}^{p}:=L_{T}^{\infty} H_{0}^{1}(\Omega) \cap L_{T}^{p} W^{1, q}(\Omega), \quad \frac{1}{p}+\frac{d}{q}=\frac{d}{2},
$$

equipped with the natural norm. The parameter $p>2$ will be chosen close enough to 2 . Unfortunately in dimensions $d \geqslant 5$ it is not evident that the transformation $K_{u_{0}}$ defined by (3.20) contracts suitable balls of $X_{T}^{p}$. However now we will show that $K_{u_{0}}$ maps a suitable ball of $X_{T}^{p}$ (of radius $c\left\|u_{0}\right\|_{H_{0}^{1}(\Omega)}$ ) into itself, for some $p>2$, provided $T$ be small enough as far as $\alpha<\frac{2}{d-2}$.

Proposition 3.4. For every $\alpha \in] 0, \frac{2}{d-2}[$ there exist $\beta \geqslant \alpha, \theta>0, p>2$ such that for $\left.T \in] 0,1\right]$,

$$
\left\|K_{u_{0}}(u)\right\|_{X_{T}^{p}} \lesssim\left\|u_{0}\right\|_{H_{0}^{1}(\Omega)}+T^{\theta}\|u\|_{X_{T}^{p}}\left(1+\|u\|_{X_{T}^{p}}^{\beta}\right) .
$$

Proof. Let $\psi \in C_{0}^{\infty}(\mathbb{R})$ be such that $\psi(x)=0$ for $|x|<1$ and $\psi(x)=1$ for $|x|>2$. Split the map $\Phi$ defined by (3.11) as $\Phi(u)=\Phi_{1}(u)+\Phi_{2}(u)$, where

$$
\left(\Phi_{1}(u)\right)(t):=\int_{0}^{t} \mathrm{e}^{i(t-\tau) \Delta_{D}}\left(\psi\left(|u(\tau)|^{2}\right) F(u(\tau))\right) \mathrm{d} \tau .
$$


Using Proposition 2.14, we deduce that for every $\alpha>0$, every $p>2$, we have

$$
\left\|\Phi_{2}(u)\right\|_{X_{T}^{p}} \lesssim\|u\|_{L_{T}^{1} H_{0}^{1}(\Omega)} \lesssim T\|u\|_{X_{T}^{p}} .
$$

Hence the issue is to evaluate $\Phi_{1}(u)$ in $X_{T}^{p}$ for some $p>2$ and $\left.\left.T \in\right] 0,1\right]$. We first prove that with a proper choice of $\beta \geqslant \alpha, \theta>0, p>2,(\tilde{p}, \tilde{q}), \tilde{p} \in[1,2[$ satisfying (2.33), one has the bound

$$
\left\|\psi\left(|u|^{2}\right) F(u)\right\|_{L_{T}^{\tilde{p}} W^{1, \tilde{q}}(\Omega)} \lesssim T^{\theta}\|u\|_{X_{T}^{p}}\left(1+\|u\|_{X_{T}^{p}}^{\beta}\right) .
$$

Since $\tilde{q} \leqslant 2$, we use the pointwise bound

$$
\left|\psi\left(|u|^{2}\right) F(u)\right| \lesssim|u|^{1+2 /(d-2)} .
$$

Therefore due to Proposition 2.1,

$$
\left\|\psi\left(|u|^{2}\right) F(u)\right\|_{L_{T}^{\tilde{p}} L^{\tilde{q}}(\Omega)} \lesssim T^{1 / \tilde{p}}\|u\|_{L_{T}^{\infty} H_{0}^{1}(\Omega)}^{d /(d-2)} \lesssim T^{1 / \tilde{p}_{\|}}\|u\|_{X_{T}^{p}}^{d /(d-2)} .
$$

Next we observe that

$$
\left|\nabla\left(\psi\left(|u|^{2}\right) F(u)\right)\right| \lesssim|u|^{\beta}|\nabla u|, \quad \beta \geqslant \alpha,
$$

and we deduce that the main point in the proof of (3.22) is to bound $|u|^{\beta} \nabla u$ in $L_{T}^{\tilde{p}} L^{\tilde{q}}(\Omega)$ for some $\beta \in\left[\alpha, \frac{2}{d-2}[\right.$ and a suitable choice of $(\tilde{p}, \tilde{q})$ satisfying (2.33). Let us first perform the chain of inequalities, involved in the estimate for $|u|^{\beta} \nabla u$,

$$
\begin{aligned}
& \left\||u|^{\beta} \nabla u\right\|_{L_{T}^{\tilde{p}} L^{\tilde{q}}(\Omega)} \lesssim\left\||u|^{\beta}\right\|_{L_{T}^{p_{1}} L^{q_{1}(\Omega)}}\|\nabla u\|_{L_{T}^{p_{2}} L^{q_{2}(\Omega)}} \\
& \lesssim\|u\|_{L_{T}^{\beta p_{1}} L^{\beta q_{1}(\Omega)}}^{\beta}\|u\|_{X_{T}^{p}} \\
& \lesssim\|u\|_{L_{T}^{\beta p_{1} W^{1, q^{\star}}(\Omega)}}^{\beta}\|u\|_{X_{T}^{p}} \\
& \lesssim T^{\theta}\|u\|_{X_{T}^{p}}^{\beta+1},
\end{aligned}
$$

where $\theta=\frac{1}{p_{1}}-\frac{\beta}{p^{\star}}$ and the following conditions are imposed on the parameters appearing in the above computation,

$$
\begin{aligned}
\frac{1}{\tilde{p}}+\frac{d}{\tilde{q}}=1+\frac{d}{2}, \quad \tilde{p} \in[1,2[ & \text { (Proposition } 2.15 \text { condition), } \\
\frac{1}{p_{1}}+\frac{1}{p_{2}}=\frac{1}{\tilde{p}}, \quad \frac{1}{q_{1}}+\frac{1}{q_{2}}=\frac{1}{\tilde{q}} & \text { (Hölder inequality condition), } \\
\frac{1}{p_{2}}+\frac{d}{q_{2}}=\frac{1}{p^{\star}}+\frac{d}{q^{\star}}=\frac{d}{2}, \quad p_{2}>2, p^{\star}>2 & \text { (Proposition } 2.14 \text { condition), } \\
\frac{1}{q^{\star}}-\frac{1}{\beta q_{1}}=\frac{1}{d}, \quad q^{\star}<d & \text { (Sobolev embedding condition), } \\
\beta p_{1}<p^{\star} & \text { (small factor condition). }
\end{aligned}
$$

Let us now show that it is indeed possible to make a proper choice of the parameters involved in the above computation. We start by fixing $\left(p_{2}, p^{\star}\right)$ as follows,

$$
\frac{1}{p_{2}}:=\frac{1}{2}-\delta, \quad \frac{1}{p^{\star}}:=\frac{1}{2}-\delta,
$$

where $\delta>0$ is a sufficiently small parameter to be fixed later. The other parameters will be chosen following the scheme,

$$
\left(p_{2}, p^{\star}\right) \rightarrow\left(q_{2}, q^{\star}\right) \rightarrow q_{1} \rightarrow \tilde{q} \rightarrow \tilde{p} \rightarrow p_{1} .
$$


In order to keep the parameters in the permitted regions, in the first step we should verify that $q^{\star}<d$, in the third one that $\frac{1}{\tilde{q}} \in\left[\frac{1}{2}, \frac{d+1}{2 d}\right]$ and in the final step we should verify what is the restriction imposed by the small factor assumption $\beta p_{1}<p^{\star}$.

Taking into account Proposition 2.14 condition, we define,

$$
\frac{1}{q_{2}}:=\frac{d-1}{2 d}+\frac{\delta}{d}, \quad \frac{1}{q^{\star}}:=\frac{d-1}{2 d}+\frac{\delta}{d} .
$$

Note that $\left.q^{\star} \in\right] 2, d[$ and taking into account Sobolev embedding restriction, we set,

$$
\frac{1}{q_{1}}:=\frac{\beta(d-3)}{2 d}+\frac{\delta \beta}{d} \text {. }
$$

Next taking into account Hölder inequality condition, we define

$$
\frac{1}{\tilde{q}}:=\frac{\beta(d-3)+d-1}{2 d}+\frac{\delta(\beta+1)}{d} .
$$

An easy computation shows that by choosing $\delta$ small enough and $\beta$ sufficiently close to $\frac{2}{d-2}$, we can have

$$
\frac{1}{\tilde{q}} \in\left[\frac{1}{2}, \frac{d+1}{2 d}[.\right.
$$

Next Proposition 2.15 condition permits us to define $\tilde{p}$ and due to (3.23) we have $\tilde{p} \in[1,2[$. Finally taking into account the first Hölder inequality condition, we set

$$
\frac{1}{p_{1}}:=1-\frac{\beta(d-3)}{2}-\delta \beta .
$$

It is now easy to verify that the small factor condition $\beta p_{1}<p^{\star}$ is equivalent to $\beta<\frac{2}{d-2}$ which ends the proof of (3.22). Using Proposition 2.15, for $p>2, p<p^{\star}$,

$$
\left\|\Phi_{1}(u)\right\|_{X_{T}^{p}} \lesssim\left\|\left(1-\Delta_{D}\right)^{1 / 2}\left(\psi\left(|u|^{2}\right) F(u)\right)\right\|_{L_{T}^{\tilde{p}} L \tilde{q}(\Omega)},
$$

where $(\tilde{p}, \tilde{q}), \tilde{p} \in[1,2[$, satisfies (2.33). Using (3.22) together with (2.1) and an approximation argument, we obtain that there exists $C>0$ such that for every $u \in X_{T}^{p}$,

$$
\left\|\left(1-\Delta_{D}\right)^{1 / 2}\left(\psi\left(|u|^{2}\right) F(u)\right)\right\|_{L_{T}^{\tilde{p}} L^{\tilde{q}}(\Omega)} \leqslant C T^{\theta}\|u\|_{X_{T}^{p}}\left(1+\|u\|_{X_{T}^{p}}^{\beta}\right),
$$

where $(\tilde{p}, \tilde{q}, p, \beta, \theta)$ are the same as in (3.22). This completes the proof of Proposition 3.4.

Due to Propositions 2.15 and 3.4, we deduce that if $\alpha<\frac{2}{d-2}$ and $u_{0} \in H_{0}^{1}(\Omega)$ then the map $K_{u_{0}}$ sends a suitable ball in $X_{T}^{p}$ into itself, provided $T \ll 1$ and $p$ close enough to 2 . Finally, using Proposition 3.1 we obtain that $K_{u_{0}}$ is a contraction on this ball in the weaker topology $L_{T}^{\infty} L^{2}(\Omega) \cap L_{T}^{p^{\star}} L^{q^{\star}}(\Omega)$, where $p^{\star}$ is the exponent $p$ involved in Proposition 3.1 and $\left(p^{\star}, q^{\star}\right)$ satisfies (2.28). This completes the proof of the existence claim of Theorem 1.

Remark 3.5. Various continuous dependence with respect to the initial data results can be obtained by using the bounds on $\left\|K_{u_{0}}\right\|_{X_{T}}$. Unfortunately these considerations do not give Lipschitz bounds in $H_{0}^{1}(\Omega)$ for the flow map.

\subsection{Lipschitz bound in four space dimensions}

In this section $d=4$. The argument, we present is inspired by the work of Keraani (see [20]) and is very similar to the consideration in [10, Appendix 1]. In order to carry out this argument one essentially needs to control 
quadratic nonlinearities in the analysis performed in the previous section. Let $B$ be a bounded convex set in $H_{0}^{1}(\Omega)$. Fix $T>0$. In the previous section, we established the existence of a well-defined flow map

$$
\Phi: u_{0} \in B \mapsto u \in C\left([-T, T], H_{0}^{1}(\Omega)\right) .
$$

Our aim is to show that the differential of $\Phi$ is bounded in the natural functional framework, a fact which clearly implies the Lipschitz property. Fix $u_{0} \in B$ and let $\Phi\left(u_{0}\right)=u$. Then for every $v_{0} \in H_{0}^{1}(\Omega)$, we have that $\Phi_{u_{0}}^{\prime}\left(v_{0}\right):=v$ satisfies the equation

$$
\left(i \partial_{t}+\Delta\right) v=\frac{\partial F}{\partial u} v+\frac{\partial F}{\partial \bar{u}} \bar{v}
$$

with $\left.v\right|_{t=0}=v_{0}$. For $0<\delta \ll 1$, we define the space

$$
Y_{T}^{\delta}:=L_{T}^{2 /(1-2 \delta)} W^{1,8 /(3+2 \delta)}(\Omega) \cap L_{T}^{3} W^{1,12 / 5}(\Omega)
$$

equipped with the natural norm. If $I \subset \mathbb{R}$ is an interval, we can define the space $Y_{I}^{\delta}$ in a natural manner. Similarly to the previous section, we introduce the space $X_{T}^{p}$ and moreover by taking $p$ close enough to 2 and $\delta$ small enough, one gets the bound

$$
\|v\|_{X_{T_{1}}^{p}} \lesssim\left\|v_{0}\right\|_{H_{0}^{1}(\Omega)}+\left(T_{1}+\|u\|_{Y_{T_{1}}^{\delta}}\right)\|v\|_{X_{T_{1}}^{p}},
$$

where $T_{1} \in[0, T]$. As a consequence there exists a positive constant $A$ such that if $T_{1}+\|u\|_{Y_{T_{1}}^{\delta}} \leqslant A$, then $\|v\|_{X_{T_{1}}^{p}} \leqslant C\left\|v_{0}\right\|_{H_{0}^{1}(\Omega)}$ for some $C>0$ and in particular

$$
\|v\|_{L_{T_{1}}^{\infty} H_{0}^{1}(\Omega)} \leqslant C\left\|v_{0}\right\|_{H_{0}^{1}(\Omega)} .
$$

Next we split $[-T, T]$ into $N$ intervals $I_{1}, \ldots, I_{N}$ such that

$$
\left|I_{k}\right|+\|u\|_{Y_{I_{k}}^{\delta}} \leqslant A
$$

A simple geometric observation shows that we can always realize the above slicing with $N$ satisfying

$$
N \lesssim T+\|u\|_{Y_{T}^{\delta}}
$$

Note that the above bound for $N$ depends only on $T$ and $u$ but not on $v_{0}$. Iterating estimate (3.25), we finally infer

$$
\|v\|_{L_{T}^{\infty} H_{0}^{1}(\Omega)} \leqslant C^{N}\left\|v_{0}\right\|_{H_{0}^{1}(\Omega)}
$$

which completes the proof of the Lipschitz bound for $d=4$.

\section{Proof of Theorem 2}

The proof of Theorem 2 has the flavor of the considerations of the previous section. We first prove that for $u_{0} \in L^{2}(\Omega)$, the map $K_{u_{0}}$ defined by (3.20) is a contraction in a suitable set of the space

$$
X_{T}^{p}:=L_{T}^{\infty} L^{2}(\Omega) \cap L_{T}^{p} L^{q}(\Omega), \quad \frac{1}{p}+\frac{d}{q}=\frac{d}{2},
$$

provided $T \ll 1$ and a suitable choice of $p>2$. Indeed that follows from the next statement.

Proposition 4.1. For every $\alpha \in] 0, \frac{2}{d}\left[\right.$ there exists $\beta \in\left[\alpha, \frac{2}{d}[\right.$ and $p>2$ such that for $\left.T \in] 0,1\right]$,

$$
\begin{aligned}
& \left\|K_{u_{0}}(u)\right\|_{X_{T}^{p}} \lesssim\left\|u_{0}\right\|_{L^{2}(\Omega)}+T^{1-\beta d / 2}\|u\|_{X_{T}^{p}}\left(1+\|u\|_{X_{T}^{p}}^{\beta}\right), \\
& \left\|K_{u_{0}}(u)-K_{u_{0}}(v)\right\|_{X_{T}^{p}} \lesssim T^{1-\beta d / 2}\|u-v\|_{X_{T}^{p}}\left(1+\|u\|_{X_{T}^{p}}^{\beta}+\|v\|_{X_{T}^{p}}^{\beta}\right) .
\end{aligned}
$$


Proof. We shall only prove (4.2) since (4.1) follows from (4.2) with $v=0$ combined with Proposition 2.14 with $s=0$. Let $\psi$ be the cut-off function used in the proof of Proposition 3.4. We write

$$
F(u)-F(v)=\psi\left(|u|^{2}+|v|^{2}\right)(F(u)-F(v))+\left(1-\psi\left(|u|^{2}+|v|^{2}\right)\right)(F(u)-F(v)),
$$

and we use Proposition 2.14 with $s=0$ and (3.4). We obtain

$$
\|\Phi(u)-\Phi(v)\|_{X_{T}^{p}} \lesssim T\|u-v\|_{L_{T}^{\infty} L^{2}(\Omega)}+\left\|(u-v)\left(|u|^{\beta}+|v|^{\beta}\right)\right\|_{L_{T}^{\tilde{p}} L^{\tilde{q}}(\Omega)},
$$

where the transformation $\Phi$ is defined in Proposition 3.2, $\beta \in\left[\alpha, \frac{2}{d}[\right.$ and the pair $(\tilde{p}, \tilde{q}), \tilde{p} \in[1,2[$, satisfies (2.33). Next, using Hölder inequality, we obtain

$$
\left\|(u-v)\left(|u|^{\beta}+|v|^{\beta}\right)\right\|_{L_{T}^{\tilde{p}} L^{\tilde{q}}(\Omega)} \lesssim\|u-v\|_{L_{T}^{p_{1} L^{q_{1}(\Omega)}}}\left(\|u\|_{L_{T}^{\beta p_{2} L^{\beta q_{2}(\Omega)}}}^{\beta}+\|v\|_{L_{T}^{\beta p_{2} L^{\beta q_{2}(\Omega)}}}^{\beta}\right),
$$

where

$$
\frac{1}{p_{1}}+\frac{1}{p_{2}}=\frac{1}{\tilde{p}}, \quad \frac{1}{q_{1}}+\frac{1}{q_{2}}=\frac{1}{\tilde{q}}, \quad \frac{1}{p_{1}}+\frac{d}{q_{1}}=\frac{d}{2} .
$$

Let $p^{\star}$ be such that

$$
\frac{1}{p^{\star}}+\frac{d}{\beta q_{2}}=\frac{d}{2} \text {. }
$$

Let us choose the parameters $\left(p_{1}, p_{2}, q_{1}, q_{2}, \tilde{p}, \tilde{q}, p^{\star}\right)$ satisfying (2.33), (4.3), (4.4) as follows,

$$
\begin{aligned}
& \frac{1}{p_{1}}=\frac{1}{p^{\star}}=\frac{1}{2}-\delta, \quad \frac{1}{q_{1}}=\frac{d-1}{2 d}+\frac{\delta}{d}, \quad \frac{1}{q_{2}}=\frac{\beta(d-1)}{2 d}+\frac{\beta \delta}{d}, \\
& \frac{1}{\tilde{q}}=\frac{(\beta+1)(d-1)}{2 d}+\frac{(\beta+1) \delta}{d}, \quad \frac{1}{\tilde{p}}=\frac{3}{2}-\frac{\beta(d-1)}{2}-(\beta+1) \delta, \\
& \frac{1}{p_{2}}=1-\frac{\beta(d-1)}{2}-\beta \delta .
\end{aligned}
$$

We choose the free parameters $\delta$ and $\beta$ as follows. Since $d \geqslant 2$ we take $\delta$ positive but small enough and $\beta$ close to $2 / d$ in order to ensure that $\tilde{p} \in[1,2[$. Thus, we finally get

$$
\|u-v\|_{L_{T}^{p_{1}} L^{q_{1}(\Omega)}} \lesssim\|u-v\|_{X_{T}}
$$

and

$$
\begin{aligned}
& \|u\|_{L_{T}^{\beta p_{2}} L^{\beta q_{2}(\Omega)}}^{\beta}+\|v\|_{L_{T}^{\beta p_{2} L^{\beta q_{2}(\Omega)}}}^{\beta} \lesssim T^{1-\beta d / 2}\left(\|u\|_{L_{T}^{p^{\star}} L^{\beta q_{2}(\Omega)}}^{\beta}+\|v\|_{L_{T}^{p^{\star} L^{\beta q_{2}(\Omega)}}}^{\beta}\right) \\
& \lesssim T^{1-\beta d / 2}\left(\|u\|_{X_{T}}^{\beta}+\|v\|_{X_{T}}^{\beta}\right) .
\end{aligned}
$$

This completes the proof of Proposition 4.1 .

We now turn to the uniqueness issue. Let first $\alpha<\frac{1}{d}$. Assume that $u$ and $v$ are two solutions both in $L_{T}^{\infty} L^{2}(\Omega)$. Then $F(u)$ and $F(v)$ are in the sum of $L_{T}^{\infty} L^{2 /(1+\alpha)}(\Omega)$ and $L_{T}^{\infty} L^{2}(\Omega)$. Since $\alpha<\frac{1}{d}$ implies $\frac{2}{1+\alpha}>\frac{2 d}{d+1}$, we can apply Propositions 2.14 and 2.15 to deduce that $u, v \in X_{T}^{p}, p>2$. The uniqueness in the considered case now follows from (4.2). Let now $\alpha \geqslant \frac{1}{d}$. Assume that $u$ and $v$ are two solutions both in $L_{T}^{\infty} L^{2}(\Omega) \cap L_{T}^{p} L^{q}(\Omega)$ with $2<p<\frac{2(\alpha+1)}{\alpha d-1}$. Then $F(u)$ and $F(v)$ belong to the sum of $L_{T}^{p /(1+\alpha)} L^{q /(1+\alpha)}(\Omega)$ and $L_{T}^{\infty} L^{2}(\Omega)$. Let $\tilde{q}:=\frac{q}{1+\alpha}$. It follows from the condition on $p$ that $\tilde{q}>\frac{2 d}{d+1}$. Moreover if $\tilde{p}$ is such that $\frac{1}{\tilde{p}}+\frac{d}{\tilde{q}}=\frac{d}{2}+1$ then $\tilde{p}<\frac{p}{1+\alpha}$ since

$$
\frac{1+\alpha}{p}+\frac{d(1+\alpha)}{q}=\frac{(1+\alpha) d}{2}<\frac{d}{2}+1=\frac{1}{\tilde{p}}+\frac{d}{\tilde{q}} .
$$


Therefore by Propositions 2.14 and 2.15, we obtain that $u$ and $v$ are both in $X_{T}^{p}, p>2$, and the uniqueness in the considered case follows as above from (4.2). This completes the proof of Theorem 2.

\section{Acknowledgements}

This research was carried out during a stay of the first author at the University of California, Berkeley, funded by the Miller Institute for Basic Research in Science. We are grateful to the referee for pointing out to us several imprecisions in the first version of the paper.

\section{References}

[1] M. Ben Artzi, S. Klainerman, Decay and regularity for the Schrödinger equation, J. Anal. Math. 58 (1992) $25-37$.

[2] J. Bergh, J. Löfstrom, Interpolation Spaces, Springer-Verlag, 1976.

[3] J. Bourgain, Global Solutions of Nonlinear Schrödinger Equations, in: Colloq. Publications, American Math. Soc., 1999.

[4] J. Bourgain, Problems in Hamiltonian PDE's. GAFA 2000 (Tel Aviv, 1999), Geom. Funct. Anal. (Special Volume, Part I) (2000) $32-56$.

[5] H. Brézis, T. Gallouet, Nonlinear Schrödinger evolution equations, Nonlinear Anal. 4 (1980) 677-681.

[6] N. Burq, Contrôle de l'équation des ondes dans des ouverts peu réguliers, Asymptotic Anal. 14 (1997) $157-191$.

[7] N. Burq, Décroissance de l'énergie locale de l'équation des ondes pour le problème extérieur et absence de résonance au voisinage du réel, Acta Math. 180 (1998) 1-29.

[8] N. Burq, Semi-classical estimates for the resolvent in nontrapping geometries, Internat. Math. Res. Notices (2002) $221-241$.

[9] N. Burq, P. Gérard, N. Tzvetkov, Strichartz inequalities and the nonlinear Schrödinger equation on compact manifolds, Amer. J. Math., in press.

[10] N. Burq, P. Gérard, N. Tzvetkov, The Cauchy problem for the nonlinear Schrödinger equation on a compact manifold, Proceedings of the Öresund Symposium on Partial Differential Equations, Lund, May 23-25, 2002, J. Nonlinear Math. Phys. 10 (2003) 12-27, Supplement 1.

[11] N. Burq, P. Gérard, N. Tzvetkov, Two singular dynamics of the nonlinear Schrödinger equation on a plane domain, Geom. Funct. Anal. 13 (2003) 1-19.

[12] N. Burq, P. Gérard, N. Tzvetkov, An example of singular dynamics for the nonlinear Schrödinger equation on bounded domains, in: F. Colombini, T. Nishitani (Eds.), Proceedings of the Conference on Hyperbolic PDEs and Related Topics, Cortona, 2002, submitted for publication.

[13] T. Cazenave, An introduction to nonlinear Schrödinger equations, second ed., Textos de Métodos Matemáticos 26 (1993).

[14] P. Constantin, J.C. Saut, Local smoothing properties of dispersive equations, J. Amer. Math. Soc. 1 (1988) $413-439$.

[15] P. Constantin, J.C. Saut, Local smoothing properties of Schrödinger equations, Indiana Univ. Math. J. 38 (1989) $791-810$.

[16] M. Christ, A. Kiselev, Maximal functions associated to filtrations, J. Funct. Anal. 179 (2001) 409-425.

[17] S.I. Doi, Smoothing effects of Schrödinger evolution groups on Riemannian manifolds, Duke Math. J. 82 (1996) 679-706.

[18] T. Kato, On nonlinear Schrödinger equations, Ann. Inst. H. Poincaré Phys. Théor. 46 (1987) 113-129.

[19] O. Kavian, A remark on the blowing up of solutions to the Cauchy problem for nonlinear Schrödinger equations, Trans. Amer. Math. Soc. 299 (1987) 193-203.

[20] S. Keraani, Étude de quelques régimes asymptotiques de l'équation de Schrödinger, PhD Thesis, Université de Paris-Sud, December 2000.

[21] P. Lax, R. Phillips, Scattering Theory, Second edition, in: Pure Appl. Math., vol. 26, Academic Press, 1989.

[22] J.-L. Lions, Quelques méthodes de résolution des équations aux dérivées partielles non linéaires, Dunod, Paris, 1969.

[23] R.B. Melrose, J. Sjöstrand, Singularities of boundary value problems I, Comm. Pure Appl. Math. 31 (1978) $593-617$.

[24] R.B. Melrose, J. Sjöstrand, Singularities of boundary value problems II, Comm. Pure Appl. Math. 35 (1982) 129-168.

[25] T. Ogawa, T. Ozawa, Trudinger type inequalities and uniqueness of weak solutions for the nonlinear Schrödinger equations, J. Math. Anal. Appl. 155 (1991) 531-540.

[26] I. Segal, Nonlinear semi-groups, Ann. Math. 78 (1963) 339-364.

[27] C. Sulem, P.L. Sulem, The Nonlinear Schrödinger Equation. Self-Focusing and Wave Collapse, in: Applied Mathematical Sciences, vol. 139, Springer-Verlag, New York, 1999.

[28] P. Sjölin, Regularity of solutions to Schrödinger equations, Duke Math. J. 55 (1987) 699-715.

[29] H. Smith, C. Sogge, On the critical semilinear wave equation outside convex obstacles, J. Amer. Math. Soc. 8 (1995) 879-916.

[30] G. Staffilani, D. Tataru, Strichartz estimates for a Schrödinger operator with nonsmooth coefficients, Comm. Partial Differential Equations 27 (2002) 1337-1372.

[31] M. Taylor, Partial Differential Equations, in: Applied Mathematical Sciences, vols. 115, 116, 117, Springer-Verlag, New York, 1996. 
[32] M. Tsutsumi, On smooth solutions to the initial-boundary value problem for the nonlinear Schrödinger equation in two space dimensions, Nonlinear Anal. 13 (1989) 1051-1056.

[33] M. Tsutsumi, On global solutions to the initial-boundary value problem for the nonlinear Schrödinger equation in exterior domains, Comm. Partial Differential Equations 16 (1991) 885-907.

[34] Y. Tsutsumi, Global solutions of the nonlinear Schrödinger equation in exterior domains, Comm. Partial Differential Equations 8 (1984) $1337-1374$.

[35] B.R. Vainberg, Asymptotic Methods in Equations of Mathematical Physics, Gordon and Breach, New York, 1988.

[36] A. Vasy, M. Zworski, Semiclassical estimates in asymptotically euclidean scattering, Comm. Math. Phys. 212 (2000) 205-217.

[37] L. Vega, Schrödinger equations: pointwise convergence to the initial data, Proc. Amer. Math. Soc. 102 (1988) 874-878.

[38] M.V. Vladimirov, On the solvability of mixed problem for a nonlinear equation of Schrödinger type, Soviet Math. Dokl. 29 (1984) 281284. 